University of Nebraska - Lincoln

DigitalCommons@University of Nebraska - Lincoln

Publications from USDA-ARS / UNL Faculty

U.S. Department of Agriculture: Agricultural

Research Service, Lincoln, Nebraska

2009

Coccinellidae as predators of mites: Stethorini in biological

control

David J. Biddinger

Pennsylvania State University

Donald D. Weber

USDA-ARS

Larry A. Hull

Pennsylvania State University

Follow this and additional works at: https://digitalcommons.unl.edu/usdaarsfacpub

Part of the Agricultural Science Commons

Biddinger, David J.; Weber, Donald D.; and Hull, Larry A., "Coccinellidae as predators of mites: Stethorini in biological control" (2009). Publications from USDA-ARS / UNL Faculty. 387.

https://digitalcommons.unl.edu/usdaarsfacpub/387

This Article is brought to you for free and open access by the U.S. Department of Agriculture: Agricultural Research Service, Lincoln, Nebraska at DigitalCommons@University of Nebraska - Lincoln. It has been accepted for inclusion in Publications from USDA-ARS / UNL Faculty by an authorized administrator of DigitalCommons@University of Nebraska - Lincoln. 
Review

\title{
Coccinellidae as predators of mites: Stethorini in biological control
}

\author{
David J. Biddinger ${ }^{\mathrm{a}, *}$, Donald C. Weber ${ }^{\mathrm{b}}$, Larry A. Hull ${ }^{\mathrm{a}}$ \\ ${ }^{a}$ Fruit Research and Extension Center, Pennsylvania State University, P.O. Box 330, 290 University Drive, Biglerville, PA 17307, USA \\ ${ }^{\mathrm{b}}$ USDA-ARS, Invasive Insect Biocontrol and Behavior Laboratory, BARC-West Building 011A, Beltsville, MD 20705, USA
}

\section{A R T I C L E I N F O}

\section{Article history}

Received 5 January 2009

Accepted 25 May 2009

Available online 2 June 2009

\section{Keywords:}

Coccinellidae

Stethorus

Acari

Acariphagous

Tetranychid

Spider mites

\begin{abstract}
A B S T R A C T
The Stethorini are unique among the Coccinellidae in specializing on mites (principally Tetranychidae) as prey. Consisting of 90 species in two genera, Stethorus and Parasthethorus, the tribe is practically cosmopolitan. The Stethorini are found in a diverse range of habitats, including many agricultural systems such as pome and stone fruits, brambles, tree nuts, citrus, avocadoes, bananas, papaya, palms, tea, cassava, maize, strawberries, vegetables, and cotton, as well as ornamental plantings, grasslands, forests, and heathlands. Tetranychid mite outbreaks became common in many agricultural systems only after World War II, when widespread use of broad-spectrum insecticides increased. Stethorini were initially appreciated only for their ability to suppress severe outbreaks of tetranychid populations. However, research on their prey searching behaviors reveals that Stethorini use visual and olfactory stimuli to locate small mite colonies in patchy distributions, and can be very effective in regulating their prey at low densities. Moreover, acariphagous coccinellids colonize mite outbreaks earlier, and consume more pest mites, than many other mite predators. Key to the use of coccinellids in conservation biological control programs is the provision of overwintering habitats and refuges from pesticides in and near cropland. When these conditions are fulfilled, Stethorini often play important roles in maintaining suppression of tetranychid populations. Examples of successful biological mite control with Stethorini include apple orchards in Pennsylvania, USA, and citrus in Asia, and the unintended disruption of a tetranychid-based biological control program for the invasive woody weed, gorse, in Australia and New Zealand. The systematics and taxonomy of this group is challenging with many cryptic species, and molecular diagnostic tools are sorely needed. How best to utilize their mite-suppressive potential in diverse settings requires better knowledge of their requirements including utilization of alternative foods, refuges for dormancy and from nonselective pesticides, and host-finding mechanisms.
\end{abstract}

(c) 2009 Elsevier Inc. All rights reserved.

\section{Introduction}

The approximately 90 worldwide species of the tribe Stethorini Dobzhansky (genera Stethorus Weise and Parasthethorus Pang and Mao) are the only specialist mite predators in the Coccinellidae. Most predaceous Coccinellidae are specialist aphid or scale feeders, but have a wide range of accepted foods that they can utilize for various life processes (Obrycki et al., 2009; Evans, 2009; Hodek and Honěk, 2009; Lundgren, 2009a,b). Entomophagous coccinellid species (or genera) such as Hippodamia convergens Guerin-Meneville, Coleomegilla maculata De Geer, Harmonia axyridis (Pallas), Olla abdominalis (Say), Adalia, Eriopus, Scymnus, and Psyllobora feed on mites, but these prey are often suboptimal for reproduction (Dean, 1957; McMurtry et al., 1970; Hodek and Honěk, 1996; Rondon et al., 2004), and these taxa are not considered to be primary predators of mites (McMurtry et al., 1970; Hodek and Honěk, 1996).

\footnotetext{
* Corresponding author. Fax: +1 7176774112.

E-mail address: djb134@psu.edu (D.J. Biddinger).
}

Within the Stethorini, adults and larvae of Stethorus and Parastethorus spp. are specialists on spider mites (Tetranychidae) and the closely related Tenuipalpididae, which are known as false spider mites or flat mites (Chazeau, 1985), both of which are important agricultural pests worldwide. Many natural enemies within the Coleoptera, Dermaptera, Diptera, Hemiptera, Neuroptera, and Thysanoptera feed on spider mites, but vary in their degree of adaptation to and preferences for this prey and in their abilities to regulate pest mite populations (Helle and Sabelis, 1985). Biological control of spider mites has centered on two groups of biological control agents, the predatory mites in the family Phytoseiidae, and various species of Stethorini (reviews by McMurtry et al., 1970; Helle and Sabelis, 1985; McMurtry and Croft, 1997).

Our understanding of what constitutes an effective mite biological control agent has changed considerably over the last 50 years. Early research focused on the abilities of acariphagous mites and coccinellids to overcome spider mite outbreaks, and on the abilities of these predators to develop resistance to pesticides applied to control primary pests in highly managed agroecosystems such as 
apple and citrus. More recently, the potential of some phytoseiid predators to regulate spider mites at low equilibrium densities has become more widely appreciated (Croft, 1990; McMurtry and Croft, 1997; Biddinger and Hull, 2005).

Various species of Stethorini have received considerable attention over the last five decades because of their potential as biological control agents of spider mites. At least 12 species of Stethorini have been imported into the US for this purpose (Gordon, 1985) and many more have been purposefully redistributed throughout the world. Chazeau (1985) summarized information on Stethorini general biology and reported that $40 \%$ of the 68 species attacked spider mites of economic importance. This review summarizes disparate sources of information on Stethorini across many different crops worldwide for the first time. We also examine the 40-year case history of Stethorus punctum punctum (LeConte) as the key component of biological mite control in Pennsylvania apple orchards, and the sustainability of using Stethorini as biological control agents in the face of changing pesticide use patterns.

\section{Overview of Stethorini}

\subsection{Taxonomic status}

Earlier works placed Stethorus sensu latu in the tribe Scymnini, but recent works placed it in the mongeneric tribe Stethorini Dobzhansky (Chazeau, 1985; Hodek and Honěk, 1996; Giorgi et al., 2009). Stethorini are unique from all other Scymninae by the convex anterior margin of the prosternum and the truncate clypeus near the antennal bases (Gordon, 1985). Recently, Ślipińksi (2007) raised the subgenus Parastethorus Pang and Mao (Gordon and Chapin, 1983) to generic status, which this review reflects. Gordon and Chapin (1983) had earlier placed the following species into what was then the subgenus Parasthethorus: Stethorus tuncatus Kapur from Malaysia, S. gutierrezi Chazeau from New Hebrides, S. nigripes Kapur and S. histrio Chazeau from Australia. Five species from Asia are now considered to be in Parastethorus as well: S. dichiapiculus Xiao, S. guangxiensis Pang and Mao, S. indira Kapur, S. malaicus Xiao, and S. yunnanensis Pang and Mao (Yu, 1996).

\subsection{Morphology and life stages}

Almost all adults of Stethorini are small (1-1.5 mm), pubescent, black with brown or yellow legs and antennae. The pubescence may be an adaptation to aid foraging within the silken webbing of some tetranychids, a feature also seen in some species of Phytoseiidae (Houck, 1985; McMurtry and Croft, 1997). Gender can be distinguished by the small notch in the 8th sternite in male beetles (smooth in females) (McMurtry et al., 1974; Biddinger, 1993). Species cannot be determined without examining the male genitalia; most species cannot be determined in female specimens. The eggs are mostly white to creamy colored elongated ellipsoids (a few species have pinkish or dark eggs) and are glued longitudinally along the mid-veins of the undersides of the leaves. Larval color differs among species, and is often useful in field identification (Khan et al., 2002; Muma, 1955b; Putman, 1955b; Pasqualini and Antropoli, 1994; Pollock and Michels, 2002, 2003, 2007; Biddinger et al., 2008a,b). There are generally four stadia. Stethorini larvae generally do not consume the entire mite as asserted by Chazeau (1985); instead they pierce the mite eggs or active stages, repeatedly regurgitate and imbibe the prey juices, leaving the crumpled exoskeleton (Cottier, 1934; Fleschner, 1950; Collyer, 1953; Robinson, 1953; Putman, 1955a; Kaylani, 1967; Houck, 1991). Pupae are uniformly dark brown or black, covered with numerous setae, and affixed to either side of the leaves (Chazeau, 1985; Biddinger, 1993).

The cryptic nature of the adult species characteristics has undoubtedly led to species misidentifications in the literature. Their superficial similarity to some species of Delphastus, Scymnus, and Telsimia probably accounts for most reports of Stethorini feeding on scale or aphids. The importance of this group in biological control in many crops throughout the world, and the difficulties in separating species, make it an excellent candidate group for systematic treatment as well as diagnostic taxonomy by molecular barcoding methods.

\subsection{Geographical distribution}

Stethorini are present throughout the world in many different climates ranging from tropical rainforests to temperate deciduous

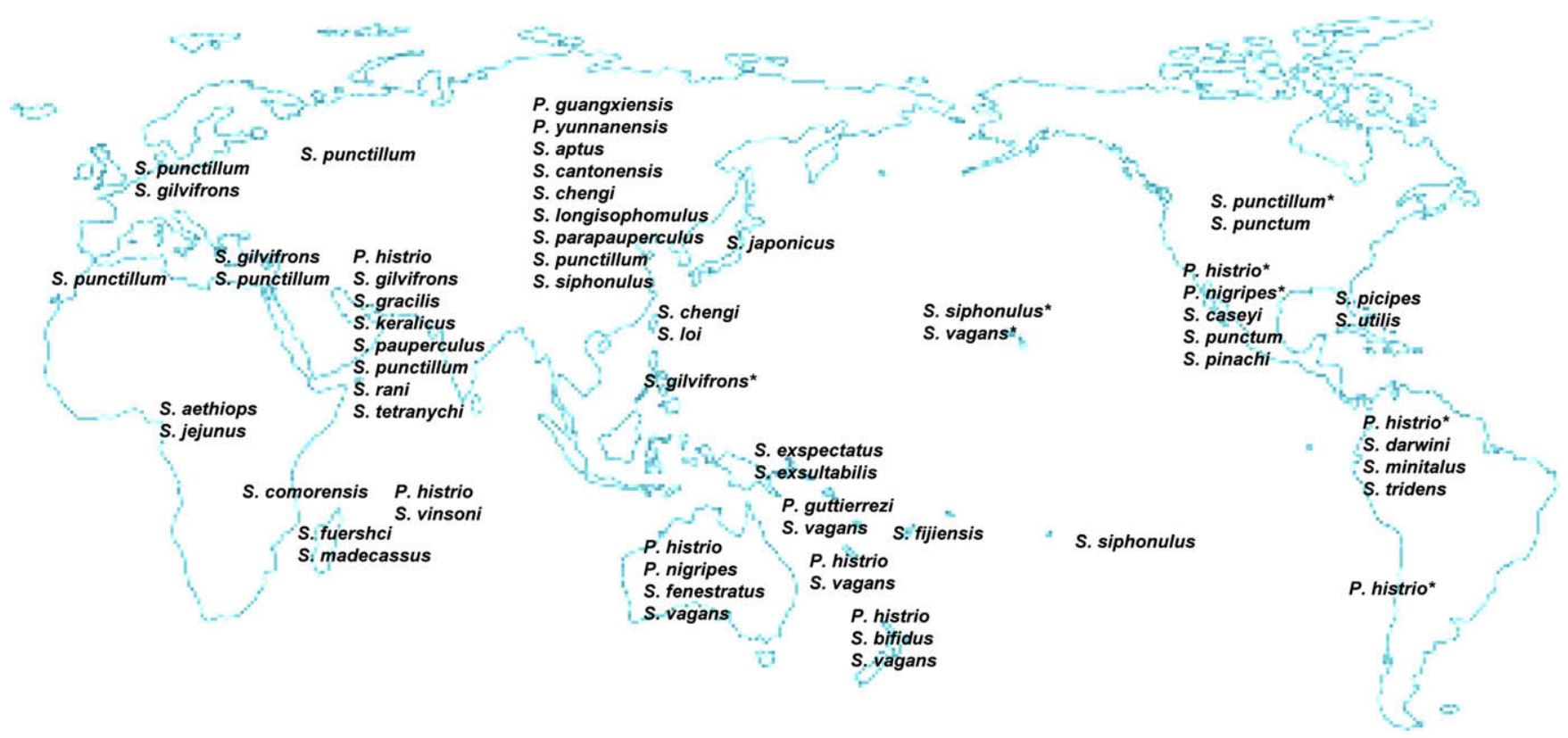

Fig. 1. Recorded geographic distribution of Stethorini on agricultural crops. * Denotes introduced or suspected introduced. See Table 1 and Kapur (1948). 
forests and plains to colder northern regions of Europe, Canada, and Russia (see map, Fig. 1, for distribution of economically important species). Stethorus punctillum Weise has the largest distribution in the group, ranging over most of temperate North America, Europe, and Asia. A major driving force behind the current geographic distribution of Stethorini has been their intentional redistribution by biological control scientists. For example, approximately 12 species (some of which were never formally identified) were introduced into California from geographically diverse areas such as Australia, China, Guatemala, India, Morocco, Pakistan, South Africa, and Turkey (Hall and Fleschner, 1958; Gordon, 1982). Some of the earliest introductions occurred in Hawaii, which apparently has no native species of Stethorini (Swezey, 1925; Fournier et al., 2002). Parastethorus nigripes (Kapur) represents the most recent introduction of Stethorini in North America (Gordon, 1993). This is an important predator of Panonychus ulmi (Koch) and Tetranychus urticae Koch in Australia (Edwards and Hodgson, 1973; Field, 1979). Parastethorus nigripes established itself in Texas corn where it consumes the Banks grass mite, Oligonychus pratensis (Banks) (Pollock and Michels, 2002), but only after introductions into California almonds failed (Hoy and Smith, 1982). The other recent addition to the North American fauna is P. histrio (=Stethorus histrio), from the Mascarene Islands east of Madagascar (Gordon and Anderson, 1979). It has since been found throughout Australia (including Tasmania), New Caledonia and parts of South America. Aguilera (1987) surmised it to be introduced accidentally into central Chile. Gordon and Chapin (1983) extended the range north into the Yucatan (Mexico), but Pollock and Michels (2003) reported it for the first time in the United States feeding on mites in corn, co-occurring with $P$. nigripes and the native species Stethorus caseyi Gordon and Chapin. Pollock and Michels (2003) found S. caseyi to be relatively scarce and speculated as to whether it had been displaced by these newly introduced species.

Additional introductions of Stethorini have likely established into new geographical ranges; the number is unknown because many species can only be identified by taxonomic experts of the group. The accidental introduction of $S$. punctillum from Europe into North America completely displaced the native S. punctum from Ontario fruit orchards and other habitats over a 20 year period (Putman, 1955a; Putman and Herne, 1966). In Québec, S. punctillum has been the only species collected from raspberry and apple crops in the past 15 years (personal communication with Michèle Roy, MAPAQ Laboratoire de Diagnostic en Phytoprotection). In Pennsylvania orchards, however, the pesticide resistant S. punctum was only found in fruit orchards, but S. punctillum was prevalent in adjacent Christmas tree plantations (Wheeler et al. 1973, Hull, personal observation).

\section{Behavior as predators}

\subsection{Prey searching behavior}

Many early studies of various species of Stethorini concluded that they are "high density predators" unable to regulate spider mite populations at low densities (Fleschner, 1950; Bailey and Caon, 1986; Tanigoshi and McMurtry, 1977). Congdon et al. (1993) challenged this "high density predator" designation and asserted that high density spider mite populations have become common only since the advent of synthetic insecticides (Huffaker et al., 1970), and that the studies that support the "high density predator" status of Stethorini ignored the spatial context of spider mite populations. While it is true that most Stethorini species do not lay eggs until mite populations are relatively high in comparison to the low mite levels maintained by some Phytoseiidae, the
Stethorini have provided economically successful regulation of pest mites in several cases. Reasons underlying these success stories include (a) the regulatory potential of the long-lived adults; (b) the ability of adults to rapidly immigrate into cropland; and (c) the ability of non-pest tetranychid mite populations, non-tetranychid mites, extrafloral nectaries, aphid honeydew, and pollen, to support populations of Stethorini before pest mite populations reach outbreak proportions in crops. The importance of Stethorini species worldwide as biological control agents is also suggested by the over 500 references found in the course of this review.

Congdon et al. (1993) hypothesized that the active discovery of rare and very small prey patches by adult Stethorini was a key element in their natural interactions with spider mites over evolutionary time. Few, if any, predator eggs are laid until mite populations become high enough to support the development of larvae, a common phenomenon in coccinellids (Seagraves, 2009). Then the impressive numerical response of Stethorini would reduce damaging mite populations later in the season on crops which are often more tolerant of late season mite injury. Pesticides applied for mite control frequently kill Stethorini, and so the natural low-density interactions between predators and pests do not occur. Subsequently, spider mite populations increase, and Stethorini immigrate in response to these ample mite populations. Thus, there is the illusion that Stethorini function as "high density predators" within pesticide-treated cropland.

Even when insecticides are applied to crops, Stethorini can quickly reduce mite populations and may prevent economic injury. Adult $S$. punctum were able to find eight $P$. ulmi-infested apple trees out of a 1-ha orchard, even though these infested trees had fewer than 1 mite/leaf (Hull et al., 1977b). Similarly, S. punctum was able to find small patches of pest mites in avocados, citrus, and raspberries (Congdon et al., 1993; McMurtry and Johnson, 1966; Haney et al., 1987). Similar low-density detection of mite prey has also been demonstrated by $S$. chengi in citrus orchards in China (Chen, 1993; Chen and Zhao, 1994). The cues that Stethorini adults employ to find these small populations of mites are poorly understood, but some evidence points to a combination of visual and olfactory cues. The detection of prey by the larval stages is generally thought to be tactile with visual cues being non-existent (Fleschner, 1950; Putman, 1955a,b; McMurtry et al., 1970; Houck, 1985; Houck, 1986).

\subsubsection{Visual stimuli}

At least some species of Stethorini are visually attracted to white or yellow colors. When spider mite populations are low, sticky traps can be more effective than visual counts in monitoring Stethorus populations (Felland et al., 1995; Biddinger and Hull, 1995; Takahashi et al., 2001a; Roy et al., 2005). Readshaw (1975) used white sticky card traps to monitor Stethorini populations in orchards early in the season. Felland et al. (1995) found that yellow sticky card traps captured up to 15 times more $S$. punctum than white traps. Spider mite feeding causes a yellowing (bronzing) of leaves, and like many other coccinellids (Seagraves, 2009), foraging Stethorini adults may use this color as an indication of prey. Stethorus punctum is primarily diurnal (Hull et al., 1977b), which reinforces the hypothesis that vision is an important foraging cue.

\subsubsection{Olfactory stimuli}

Prey-derived olfactory stimuli are another important cue for foraging Stethorini adults. Colburn and Asquith (1970) examined the olfactory response of adult $S$. punctum to $P$. ulmi under choice conditions. The beetles walked preferentially toward prey versus the no-prey treatments in the presence and absence of apple foliage. Later work found that $S$. japonicus and S. punctillum also are attracted to the volatiles of tetranychid-infested plants 
(unpublished data cited in Takahashi et al., 2001b; Lentz et al., 2004). Lentz et al. (2004) went on to show that at least some of the attractive volatiles were produced by infested cucumber and bean plants.

There have been several efforts to identify the prey-associated chemistry that is attractive to Stethorini. James (2003a) and James and Price (2004) found that S. punctum was attracted to white traps baited with a synthetic herbivore-induced plant volatiles including methyl salicylate and (Z)-3-hexenyl acetate under field conditions. More $S$. punctum were recovered from beating samples in hop yards treated with time-release methyl salicylate, and the attraction of this and other predators was observed to reduce $T$. urticae numbers dramatically in an unreplicated study. Similar results were obtained with unbaited sticky cards in replicated vineyards treated with time-release methyl salicylate. James (2005) also found cis-3-hexen-1-ol and benzaldehyde to be attractive to S. punctum in additional field trials with multiple synthetic plant volatiles.

Although it is clear that olfactory cues are important in attracting foraging Stethorini, beetles do not necessarily respond to specific olfactory stimuli under field conditions. For example, S. japonicus was not significantly attracted to sticky traps with plant volatile blends in a Japanese pear orchard, perhaps because high Tetranychus kanzawai Kishida populations may have obscured the trap-associated volatile attractants (Takahashi et al., 2001a). In another study, infested host odors or other plant cues (e.g., plant location or stature) may have inhibited the attractiveness of mite-infested bean plants to $S$. japonicus (Takahashi et al., 2001b). Gillespie et al. (1997) used potted bean plants infested with T. urticae to collect S. punctillum and several other mite predators in several habitats in British Columbia, Canada, indicating perhaps that not all Stethorini spp. are equally attracted to the same volatiles.

\subsection{Trophic ecology and life history parameters}

\subsubsection{Relative quality of mites as prey}

As shown in Table 1, Stethorini attack a large number of tetranychid species on many different crops. Although it appears that most feed on multiple tetranychid prey species, some species are more specialized than others and some tetranychid prey are less suitable than others. For example, S. punctillum and S. gilvifrons do not readily feed on or reproduce on the tetranychid mites of the genus Bryobia (Putman, 1955a; Kaylani, 1967). Houck and Strauss (1985) found a weak, but consistent, feeding preference of $S$. punctum for T. urticae over P. ulmi, but this preference was affected by preconditioning of the predator for specific mite species, and preferences did not persist from larval to adult stages.

Many prey change in their suitability for coccinellids as they age (Hodek and Honěk, 2009), and mites are no exception. Stethorini adults and larvae often prefer mite eggs over other pest life stages (Houck, 1991; Tanigoshi and McMurtry, 1977), but S. madecassus preferred adult mites over eggs as prey (Chazeau, 1974b). Generally, eggs are the most nutritious life stage of a prey item, largely because they contain less water (Lundgren, 2009a). Also, the eggs may be less defended, more apparent to mobile adults, or more abundant than other life stages. Most studies employ only a single life stage of mite prey (usually adult females or eggs) when they determine the developmental rates of various Stethorini species, and do not examine the nutritional values of the various prey stages or intraspecific competition between predator stages. Houck (1991) speculated that varying the mite stages consumed by $S$. punctum might prevent severe oscillations in both the predator and the prey populations. As with many other coccinellids, Stethorini females often consume more prey than conspecific males (Hull et al., 1977b; Chazeau, 1985).
Some Stethorini utilize non-tetranychid mites as alternative prey in times of starvation. Ullah (2000) compared the suitability of the phytoseiid predatory mite, Phytoseiulus persimilis AthiasHenriot, the eriophyid rust mite, Auclops lycopersi (Massee), the tenupalpid broad mite, Polyphagotarsonemus latus (Banks), and the tetranychid spider mite, T. urticae as prey for Stethorus vagans (Blackburn) adults. Mating and oviposition of fertile eggs was observed only in S. vagans adults that were fed tetranychid or broad mites. However, mating, oviposition and fecundity were much lower with the broad mite diet than with tetranychid prey. Rust mite, but not phytoseiid mite, diets prolonged survival. Hull (personal observation) has observed S. punctum commonly feeding on both the phytoseiid (most likely Neoseiulus fallacis (Garman)) and the eriophyid mite, Aculus schlechtendali (Nalepa) early in the season when spider mite populations were very low and the predators were pre-reproductive. Kaylani (1967) and Putman (1955a) observed Stethorus spp. feeding on phytoseiids in the field, and Mathur (1969) observed adult S. gilvifrons eating a predatory Anystis sp. (Acari: Anystidae).

\subsubsection{Non-mite foods of Stethorini}

Reports of Stethorini feeding on non-acarid prey and non-prey foods challenge the assertion that all species feed and develop only on spider mites. Al-Duhawi et al. (2006) found S. gilvifrons adults and larvae to be very efficient predators of all stages of the Bemisia tabaci (Gennadius) and, in laboratory trials, found the larvae completed their development on this prey alone. Silva and Bonani (2008) reported S. minutulus Gordon and Chapin feeding on the tobacco whitefly on cotton in Brazil as well. However, Silva (personal communication) confirmed that the coccinellid found feeding on whiteflies was actually a species of Delphastus (Sticholotidinae); some specimens of $S$. minutulus feeding on mites in the same whitefly colony had been sent for identification by mistake. Mathur (1969) observed that starving larval and adult S. gilvifrons ate Macrosiphum sp. (Hemiptera: Aphididae) "to a very limited extent," and Almatni and Khalil (2008) reported this species "fed occasionally" on Brachycaudus amygdalinus (Schouteden) (Hemiptera: Aphididae). Massee (1940) noted S. punctillum as a predator of Phorodon humuli (Schrank) (Hemiptera: Aphididae).

Numerous coccinellid species can utilize pollen, fungal spores, and nectar as alternative food sources (Lundgren, 2009a,b). Ullah (2000) found that addition of pollen or honey to a water-only diet doubled the longevity of adult $S$. vagans, but beetles did not mate or reproduce on either of these foods. Mathur (1969) found that S. gilvifrons consumed aphid honeydew in the lab, and also stated that the larvae "remain adhere [sic] to the sticky secretion." Pemberton and Vandenberg (1993) reported that Stethorus spp. fed at Prunus padus L. foliar extrafloral nectaries in South Korea, and Putman (1955a, 1963) found that S. punctillum, in the absence of other food, would eat raisins, aphids, aphid honeydew, and peach leaf extrafloral nectar. While all of these foods prolonged adult survival in S. punctillum, egg production was greatly reduced or prevented (Putman, 1955a).

\subsubsection{Developmental and prey consumption rates}

Some attractive characteristics of Stethorini for mite biological control are their prey consumption, longevity and high reproductive capacity (Table 2). Each adult female may consume 30-60 mites per day. Total fecundity ranges from 123 eggs in S. tridens (Fiaboe et al., 2007), 184 eggs in S. madecassus (Chazeau, 1974a, b), 221 in S. punctum (Tanigoshi and McMurtry, 1977), 279 in $S$. punctillum (Roy et al., 2003), to a high of 501 eggs in S. japonicus (Mori et al., 2005). Developmental times for most species are approximately $17 \mathrm{~d}$ at $25^{\circ} \mathrm{C}$ from oviposition to adult eclosion (Fiaboe et al., 2007; Mori et al., 2005; Putman, 1955a; Roy et al., 
Table 1

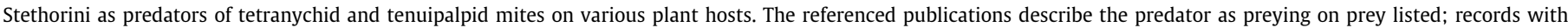
"Stethorus sp." are not listed.

\begin{tabular}{|c|c|c|c|}
\hline Predator and prey & Crop or plant & Region & Reference \\
\hline \multicolumn{4}{|l|}{ Parastethorus guangxiensis (Pang and Mao) ${ }^{\mathrm{a}}$} \\
\hline Panonychus citri (McGregor) & Citrus & China (Guangxi) & Li et al. (1990) \\
\hline \multicolumn{4}{|l|}{ Parastethorus gutierrezi (Chazeau) ${ }^{\mathrm{a}}$} \\
\hline Oligonychus sp. & Coconut palm & New Hebrides & Chazeau (1979) \\
\hline \multicolumn{4}{|l|}{ Parastethorus histrio (Chazeau) ${ }^{\mathrm{b}}$} \\
\hline Brevipalpus chilensis Baker & Beans & Chile & Aguilera (1987) \\
\hline Brevipalpus chilensis Baker & Grape & Chile & Prado (1991) \\
\hline Eutetranychus orientalis (Klein) & Citrus & India & Dhooria (1981) \\
\hline Oligonychus thelytokus Gutierrez & Lychee, Plumeria & New Caledonia & Chazeau (1979) \\
\hline Oligonychus vitis Zaher and Shehata & Table Grapes & Chile & Prado (1991) \\
\hline Oligonychus yothersi (McGregor) & Avocado & Chile & Prado (1991) \\
\hline Oligonychus sp. & Pine tree (Pinus sp.) & Australia & Houston (1980) \\
\hline Panonychus citri (McGregor) & Citrus & Chile & Aguilera (1987) \\
\hline Panonychus citri (McGregor) & Citrus & Peru & Guanilo and Martínez (2007) \\
\hline Panonychus ulmi (Koch) & Apple & Chile & Aguilera (1987) \\
\hline Tetranychus kanzawai Kishida & Bindweed (Convolvulus sp.) & Australia & Houston (1980) \\
\hline Tetranychus lintearius Dufour & Gorse & Australia & Ireson et al. (2003) \\
\hline Tetranychus neocaledonicus Andre & - & New Caledonia & Chazeau (1979) \\
\hline Tetranychus urticae Koch & Various vegetables & Reunion & Chazeau et al. (1974) \\
\hline Tetranychus urticae Koch & Papaya & Australia & Houston (1980) \\
\hline Tetranychidae & Apple & New Zealand & Chazeau (1979) \\
\hline Tetranychidae & Maize, redbud tree (Cercis) & USA (Texas) & Pollock and Michels (2003) \\
\hline \multicolumn{4}{|l|}{ Parastethorus indira (Kapur) ${ }^{a}$} \\
\hline Tetranychidae & Taro & India & Kapur (1950) \\
\hline \multicolumn{4}{|l|}{ Parastethorus nigripes (Kapur) ${ }^{\mathrm{a}}$} \\
\hline Oligonyches pratensis (Banks) & Corn & $\begin{array}{l}\text { USA (Texas, } \\
\text { Oklahoma) }\end{array}$ & Pollock and Michels (2002) \\
\hline Panonychus citri (McGregor) & Citrus & Australia & Beattie and Gellatley (1983) \\
\hline Panonychus ulmi (Koch) $)^{\mathrm{C}}$ & Apple & Australia & Walters (1976a) \\
\hline Tetranychus lambi Pritchard and Baker ${ }^{\mathrm{c}}$ & Banana & Australia & Houston (1980) \\
\hline Tetranychus urticae Koch & Apple & Australia & Edwards and Hodgson (1973) \\
\hline Tetranychus urticae Koch $^{c}$ & Soya & Australia & Houston (1980) \\
\hline Tetranychus urticae Koch & Alfalfa seed crop & Australia & Bailey and Caon (1986) \\
\hline \multicolumn{4}{|l|}{ Stethorus aethiops Weise } \\
\hline Mononychellus tanajoa (Bondar) complex & Cassava & Kenya & Yaseen et al. (1982) \\
\hline Tetranychus lombardinii Baker and Pritchard & Cassava & Kenya & Yaseen et al. (1982) \\
\hline Tetranychus neocaledonicus Andre & Cassava & Kenya & Yaseen et al. (1982) \\
\hline Tetranychus urticae Koch & Cassava & Kenya & Yaseen et al. (1982) \\
\hline \multicolumn{4}{|l|}{ Stethorus aptus Kapur } \\
\hline Panonychus citri (McGregor) & Citrus & China (Guangxi) & Li et al. (1990) \\
\hline \multicolumn{4}{|l|}{ Stethorus bifidus Kapur } \\
\hline Bryobia sp. & Apple & New Zealand & McMurtry et al. (1970) \\
\hline Panonychus ulmi (Koch), Tetranychus urticae Koch & Apple, plum, pear & New Zealand & Collyer (1964) \\
\hline Tetranychus lambi Pritchard and Baker & Apple & New Zealand & McMurtry et al. (1970) \\
\hline Tetranychus lintearius Dufour & Gorse & New Zealand & Peterson et al. (1994) \\
\hline Tetranychus urticae Koch & Raspberry & New Zealand & $\begin{array}{l}\text { Thomas and Burnip (1984), } \\
\text { Charles et al. (1985) }\end{array}$ \\
\hline \multicolumn{4}{|l|}{ Stethorus caseyi Gordon and Chapin } \\
\hline Oligonychus pratensis (Banks) & Maize & USA (Texas) & Pollock and Michels (2003) \\
\hline \multicolumn{4}{|l|}{ Stethorus chengi Sasaji } \\
\hline Panonychus citri (McGregor) & Citrus & China & Chen and Zhao (1994) \\
\hline Tetranychus urticae Koch & Papaya & Taiwan & Wen and Lee (1981) \\
\hline \multicolumn{4}{|l|}{ Stethorus comoriensis Chazeau } \\
\hline Oligonychus coffeae (Nietner) & Plumeria & Comoro Islands & Chazeau (1971b) \\
\hline Tetranychus neocaledonicus Andre & Breadfruit & Comoro Islands & Chazeau (1971b) \\
\hline \multicolumn{4}{|l|}{ Stethorus darwini (Brethes) ${ }^{\mathrm{d}}$} \\
\hline $\begin{array}{l}\text { Mononychellus caribbeanae McG., Mononychellus tanajoa (Bondar) } \\
\text { complex, various Tetranychidae }\end{array}$ & Cassava & Guyana, Surinam & Yaseen et al. (1982) \\
\hline Panonychus ulmi Koch & Apple & Brazil & Lorenzato (1987) \\
\hline Tetranychus evansi Baker and Pritchard & Tomato & Brazil & Paschoal (1970) \\
\hline \multicolumn{4}{|l|}{ Stethorus exspectatus Chazeau } \\
\hline Oligonychus, Panonychus, Schizotetranychus spp. & Various crops & New Guinea & Chazeau (1983) \\
\hline Tetranychus fijiensis Hirst & Coconut palm & New Guinea & Chazeau (1983) \\
\hline Tetranychus lambi Pritchard and Baker & Cassava & New Guinea & Chazeau (1983) \\
\hline \multicolumn{4}{|l|}{ Stethorus exsultabilis Chazeau } \\
\hline Oligonychus, Panonychus, Schizotetranychus spp. & Various crops & New Guinea & Chazeau (1983) \\
\hline Tetranychus fijiensis Hirst & Coconut palm & New Guinea & Chazeau (1983) \\
\hline Tetranychus lambi Pritchard and Baker & Cassava & New Guinea & Chazeau (1983) \\
\hline
\end{tabular}


Table 1 (continued)

\begin{tabular}{|c|c|c|c|}
\hline Predator and prey & Crop or plant & Region & Reference \\
\hline \multicolumn{4}{|l|}{ Stethorus fenestralis Houston } \\
\hline Tetranychus kanzawai Kishida & Bindweed (Convolvulus sp.) & Australia & Houston (1980) \\
\hline Tetraynchus lambi Pritchard and Baker & Banana, papaya & Australia & Houston (1980) \\
\hline Tetranychus urticae Koch & Papaya & Australia & Houston (1980) \\
\hline \multicolumn{4}{|l|}{ Stethorus fijiensis Kapur } \\
\hline Tetranychidae & - & Fiji & Swaine (1971) \\
\hline \multicolumn{4}{|l|}{ Stethorus fuerschi Chazeau } \\
\hline Oligonychus chazeaui Gutierrez & Palm tree & Madagascar & Chazeau (1971b) \\
\hline Tetranychus roseus Gutierrez & Palm tree & Madagascar & Chazeau (1971b) \\
\hline \multicolumn{4}{|l|}{ Stethorus gilvifrons (Mulsant) } \\
\hline Eutetranychus hirsti Pritchard and Baker & - & Iran & Afshari et al. (2007) \\
\hline Eutetranychus orientalis (Klein) & - & Iran & Afshari et al. (2007) \\
\hline Oligonychus afrasiaticus (McGregor) & - & Iran & Afshari et al. (2007) \\
\hline Oligonychus coffeae (Nietner) & Tea & India & Sarmah and Bhattacharyya 2002. \\
\hline Oligonychus sacchari (McGregor) & Sugarcane & Iran & Afshari (1999) \\
\hline Panonychus citri (McGregor), Eotetranychus sp. & Orange, apple, rose & Philippines & Handoko (2004) \\
\hline Panonychus ulmi Koch & Apple & Iran & Haji-Zadeh et al. (1993) \\
\hline Tetranychus turkestani Ugarov and Nikolski & Strawberry & Iran & Ahmed and Ahmed (1989) \\
\hline Tetranychus turkestani Ugarov and Nikolski & Various ornamentals & Iran & Sohrabi and Shishenbor (2007) \\
\hline Tetranychus urticae Koch & Castor bean & India & Mathur (1969) \\
\hline Tetranychus urticae Koch & Cucumbers, beans & Iran & Mehr-Khou et al. (2008) \\
\hline Tetranychidae & Various crops & Lebanon & McMurtry et al. (1970) \\
\hline \multicolumn{4}{|l|}{ Stethorus griseus Whitehead } \\
\hline Tetranychidae & Apple & New Zealand & Chazeau (1979) \\
\hline \multicolumn{4}{|l|}{ Stethorus japonicus Kamiya } \\
\hline Panonychus citri (McGregor) & Citrus & Japan & McMurtry et al. (1970) \\
\hline Panonychus citri (McGregor) & Mandarin orange & Japan & Tsuchiya (2005) \\
\hline Panonychus mori Yokoyama & Japanese pear & Japan & Kishimoto and Adachi (2008) \\
\hline Tetranychus kanzawai Kishida & Tea, hydrangea & Japan & Mori et al. (2005) \\
\hline Tetranychus urticae Koch & Apple, citrus & Japan & Mori et al. (2005) \\
\hline Tetranychus urticae Koch & Japanese pear & Japan & Kishimoto and Adachi (2008) \\
\hline Tetranychus viennenis Zacher & Japanese pear & Japan & Kishimoto and Adachi (2008) \\
\hline \multicolumn{4}{|l|}{ Stethorus jejunus Casey } \\
\hline Mononychellus tanajoa (Bondar) complex & Cassava & Kenya & Yaseen et al. (1982) \\
\hline Tetranychus lombardinii Baker and Pritchard & Cassava & Kenya & Yaseen et al. (1982) \\
\hline Tetranychus neocaledonicus Andre & Cassava & Kenya & Yaseen et al. (1982) \\
\hline Tetranychus urticae Koch & Cassava & Kenya & Yaseen et al. (1982) \\
\hline \multicolumn{4}{|l|}{ Stethorus keralicus Kapur } \\
\hline Raoiella indica Hirst (Tenuipalpidae) & Arecanut palm, coconut & India & $\begin{array}{l}\text { Puttaswamy and Rangaswamy } \\
\text { (1976) }\end{array}$ \\
\hline \multicolumn{4}{|l|}{ Stethorus loi Sasaji } \\
\hline Panonychus citri (McGregor) & Jujube & Taiwan & Wen et al. (1993) \\
\hline Tetranychus urticae Koch & Papaya & Taiwan & Wen and Lee (1981) \\
\hline Tetranychidae & Carambola & Taiwan & Chang and Leu (1986) \\
\hline \multicolumn{4}{|l|}{ Stethorus madecassus Chazeau } \\
\hline Tetranychus neocaledonicus Andre & Cotton & Madagascar & Chazeau (1971a) \\
\hline Tetranychus spp., Oligonychus spp. & Various crops & Madagascar & Chazeau (1971a) \\
\hline \multicolumn{4}{|c|}{ Stethorus parcempunctatus Puttarudrian and ChannaBasavanna } \\
\hline Raoiella indica Hirst (Tenuipalpidae) & Coconut palm & India & Gupta (2001) \\
\hline \multicolumn{4}{|l|}{ Stethorus parapauperculus Pang } \\
\hline Tetranychus piercei McGregor & Banana & China (Hainan) & Chen et al. (2005) \\
\hline Tetranychus urticae Koch & Cassava & China & Lin and Chen (1984) \\
\hline Stethorus pauperculus Weise & & & \\
\hline Oligonychus indicus (Hirst) & Sorghum & India & Kapur (1948) \\
\hline Oligonychus neocaledonicus Andre & Papaya, castor bean, and & India & Puttaswamy and \\
\hline & Various crops & & ChannaBasavanna (1977) \\
\hline Tetranychus ludeni Zacher & Eggplant & India & Puttaswamy and \\
\hline & & & ChannaBasavanna (1980) \\
\hline Tetranychus ludeni Zacher & Waterhyacinth & India & Ansari and Pawar (1992) \\
\hline Stethorus punctillum Weise & & & \\
\hline Eotetranychus buxi (Garman) & Boxwood (Buxus sp.) & USA (Maryland) & Creary (2009) \\
\hline Eotetranychus carpini (Oudemans) & Grapes & Italy & Laffi (1982) \\
\hline Eotetranychus tiliarium Hermann & Linden trees (Tilia spp.) & Germany & Jäckel et al. (2000) \\
\hline Oligonychus afrasiaticus (McGregor) & Date palm & Algeria & Idder and Pintureau (2008) \\
\hline Oligonychus bicolor (Banks) & Chestnut & Italy & Cinti et al. (1995) \\
\hline Oligonychus ununguis (Jacobi) & Chinese Chestnut & Canada & Putman (1955a) \\
\hline Oligonychus ununguis (Jacobi) & Pines, cedars and Arborvitae & USA (Pennsylvania) & Wheeler et al. (1973) \\
\hline Panonychus citri (McGregor) & Citrus & China & Tian (1995) \\
\hline Panonychus ulmi (Koch) & Fruit trees & Canada & Putman (1955a) \\
\hline Panonychus ulmi (Koch) & Fruit trees & Europe & $\begin{array}{l}\text { McMurtry et al. (1970) } \\
\text { (continued on next }\end{array}$ \\
\hline
\end{tabular}


Table 1 (continued)

\begin{tabular}{|c|c|c|c|}
\hline Predator and prey & Crop or plant & Region & Reference \\
\hline Panonychus ulmi (Koch) & Apple & Italy & Pasqualini and Antropoli (1994) \\
\hline Tetranychus bioculatus (Wood-Mason) & Marigold & Bangladesh & Taleb and Sardar (2007) \\
\hline Tetranychus cinnabarinus (Boisduval) & Cotton, apple, watermelon & Israel & Plaut (1965) \\
\hline Tetranychus mcdanieli McGregor & Raspberry & Canada & Roy et al. (2002) \\
\hline Tetranychus shoenei McGregor & Elm (Ulmus americana) & USA (Maryland) & Creary (2009) \\
\hline Tetranychus urticae Koch & Beans & Canada & Putman (1955a) \\
\hline Tetranychus urticae Koch & Sugar beets & Israel & Plaut (1965) \\
\hline Tetranychus urticae Koch & Greenhouse crops & The Netherlands & McMurtry et al. (1970) \\
\hline Tetranychus viennensis Zacher & Apple and other fruits & Turkey & Yigit and Uygun (1986) \\
\hline \multicolumn{4}{|l|}{ Stethorus punctum picipes Casey } \\
\hline Oligonychus punicae (Hirst) & Avocado & USA (California) & McMurtry et al. (1969) \\
\hline Panonychus citri (McGregor) & Citrus & USA (California) & McMurtry et al. (1970) \\
\hline Tetranychus tumidus Banks & Banana & Cuba & Perez et al. (2004) \\
\hline Tetranychidae & Walnuts, melon, apple & USA & McMurtry et al. (1970) \\
\hline \multicolumn{4}{|l|}{ Stethorus punctum punctum (LeConte) } \\
\hline Panonychus ulmi (Koch) and other tetranychids & Fruit trees & North America & McMurtry et al. (1970) \\
\hline Tetranychus mcdanieli McGregor & Fruit trees & Canada & Robinson (1953) \\
\hline Tetranychus pacificus McGregor & Fruit trees & Canada & Robinson (1953) \\
\hline \multicolumn{4}{|l|}{ Stethorus siphonulus Kapur } \\
\hline Eutetranychus banksi McGregor & Ornamentals & Hawaii & Raros and Haramoto (1974) \\
\hline Oligonychus exsiccator (Zehntner) & Sugar cane & Hawaii & Raros and Haramoto (1974) \\
\hline Panonychus citri (McGregor) & Citrus & China (Fujian) & Huang et al. (1988) \\
\hline Tetranychus cinnabarinus (Boisduval) & Papaya & Hawaii & Rosenheim et al. (2004b) \\
\hline Tetranychus neocaledonicus Andre & Custardapple (Anona sp.) & French Polynesia & Chazeau (1979) \\
\hline Tetranychus piercei McGregor & Papaya & China (Guangdong) & Lui and Lui (1986) \\
\hline Tetranychus tumidus Banks & Papaya & Hawaii & Raros and Haramoto (1974) \\
\hline Tetranychus urticae Koch & Papaya & Hawaii & Raros and Haramoto (1974) \\
\hline \multicolumn{4}{|l|}{ Stethorus tridens Gordon } \\
\hline Panonychus citri McGregor & Citrus & Peru & Guanilo and Martínez (2007) \\
\hline Tetranychus cinnabarinus (Boisduval) & Cassava & Colombia & Gordon (1982) \\
\hline Tetranychus evansi Baker and Pritchard & Tomato & Brazil & Britto et al. (2009) \\
\hline Tetranychus urticae Koch & Cassava & Colombia & Gordon (1982) \\
\hline Tetranychidae & Eggplant & Colombia & Gordon (1982) \\
\hline \multicolumn{4}{|l|}{ Stethorus utilis Horn ${ }^{\mathrm{f}}$} \\
\hline Eotetranychus hicoriae (McGregor) & Pecan & USA (Southeast) & Tedders (1983) \\
\hline Eotetranychus sexmaculatus (Riley) & Citrus & USA (Florida) & McMurtry et al. (1970) \\
\hline Eutetranychus banksi (McGregor) & Citrus & USA (Texas) & McMurtry et al. (1970) \\
\hline $\begin{array}{l}\text { Mononychellus caribbeanae (McGregror), Mononychellus tanajoa (Bondar) } \\
\text { complex, various Tetranychidae }\end{array}$ & Cassava & $\begin{array}{l}\text { Colombia, Nicaragua, } \\
\text { Trinidad }\end{array}$ & Yaseen et al. (1982) \\
\hline Oligonychus pratensis (Banks) & Sorghum & USA (Texas) & Ehler (1974) \\
\hline Tetranychus urticae Koch, Panonychus citri (McGregor) & Citrus & Cuba & Mora Morin (1991) \\
\hline Tetranychidae & Lychee & USA (Florida) & Butcher (1951) \\
\hline \multicolumn{4}{|l|}{ Stethorus vagans (Blackburn) } \\
\hline Bryobia praetiosa Koch & Clover & Australia (Tasmania) & Evans (1943) \\
\hline Oligonychus exsiccator (Zehntner) & Sugar cane & USA (Hawaii) & Swezey (1923) \\
\hline Oligonychus thelytokus Gutierrez & Lychee & New Caledonia & Chazeau (1979) \\
\hline Oligonychus sp. & Coconut palm & New Caledonia & Chazeau (1979) \\
\hline Oligonychus sp. & Pine tree (Pinus sp.) & Australia & Houston (1980) \\
\hline Panonychus ulmi (Koch) & Apple & Australia & Walters (1976a) \\
\hline Tetranychus lambi Pritchard and Baker & Cassava & New Caledonia & Chazeau (1979) \\
\hline Tetranychus lambi Pritchard and Baker & Papaya & Australia & Houston (1980) \\
\hline Tetranychus marianae McGregor & Castor bean & New Caledonia & Chazeau (1979) \\
\hline Tetranychus marianae McGregor & Ornamentals & New Hebrides & Chazeau (1979) \\
\hline Tetranychys neocaledonicus Andre & Cassava & New Caledonia & Chazeau (1979) \\
\hline Tetranychus urticae Koch & Vegetables & New Caledonia & Chazeau (1979) \\
\hline Tetranychus urticae Koch & Soya and beans & Australia & Houston (1980) \\
\hline \multicolumn{4}{|l|}{ Stethorus vinsoni Kapur } \\
\hline Tetranychus evansi Baker and Pritchard & Solanaceous plants & Mauritius & Mouitia (1958) \\
\hline
\end{tabular}

a Syn.: Stethorus, Parastethorus gen. nov. (Ślipiński, 2007).

b Syn.: Stethorus incompletus Whitehead, Stethorus histrio Chazeau (Ślipiński 2007).

c Reported as fed upon by Syn.: Stethorus loxtoni Britton and Lee.

d Syn.: Stethorus ogloblini Nunenmacher (Gordon, 1982).

e Syn.: Stethorus picipes Casey (Gordon, 1985).

f Syn.: Stethorus atomus Casey (Gordon, 1985).

2002; Tanigoshi and McMurtry, 1977). The number of Stethorini generations per year varies from 2 to 3 in temperate regions for S. punctillum and S. punctum (Putman, 1955a; Colburn and Asquith, 1971) to more than 15 per year for tropical species such as S. siphonulus and S. pauperculus (Puttaswamy and Rangaswamy, 1976; Puttaswamy and ChannaBasavanna 1977). Adult longevity is not known for most species, but appears to be longer in temperate species which undergo diapause than in tropical species, and is temperature dependent. Putman (1955a) found S. punctillum females in Canada could commonly survive and lay eggs over multiple seasons under field conditions, with an average longevity of over 400 days; males generally die sooner than females. Ullah 
Table 2

Some biological parameters observed for six species of Stethorus predators of tetranychid mites (mean/range in italics).

\begin{tabular}{|c|c|c|c|c|c|c|}
\hline $\begin{array}{l}\text { Predator } \\
\text { Prey }\end{array}$ & $\begin{array}{l}\text { S. punctillum } \\
\text { Weise, on } \\
\text { T. mcdanieli } \\
\text { McGregor } \\
\text { Roy et al. } \\
(2002,2003)\end{array}$ & $\begin{array}{l}\text { S.punctum picipes } \\
\text { Casey, on } \\
\text { O. punicae } \\
\text { (Hirst) } \\
\text { Tanigoshi and } \\
\text { McMurtry } 1977\end{array}$ & $\begin{array}{l}\text { S. madecassus } \\
\text { Chazeau, on } \\
T . \text { neocaledonicus } \\
\text { Andre } \\
\text { Chazeau }(1974 \mathrm{a}, \mathrm{b})\end{array}$ & $\begin{array}{l}\text { S. tridens } \\
\text { Gordon, on } \\
\text { T. evansi } \\
\text { Baker and Pritchard } \\
\text { Fiaboe et al. } 2007\end{array}$ & $\begin{array}{l}\text { S. japonicus } \\
\text { Kamija, on } \\
\text { T. urticae Koch } \\
\text { Mori et al. 2005; } \\
\text { Gotoh et al. } 2004\end{array}$ & $\begin{array}{l}\text { S. loi } \\
\text { Sasaji, on } \\
\text { T. kanzawai } \\
\text { Kishida } \\
\text { Shih et al. } 1991\end{array}$ \\
\hline Temperature $\left({ }^{\circ} \mathrm{C}\right)$ & $24 / 16-32$ & $24.5 / 22-27$ & $25 / 20-28.4$ & $27 / 20-30$ & $25 / 20-30$ & 24 \\
\hline Development time (days) & $17 / 49-12$ & $17 / 15.8-19$ & $14 / 11-21$ & $16 / 24-12$ & $17 / 28-11$ & 15.3 \\
\hline Length of pre-oviposition period (days) & $1.2 / 4.0-0.8$ & $5.6 / 4-6$ & $4.2 / 3-7$ & 10.3 & $4.7 / 7-13$ & 4.1 \\
\hline Total progeny (eggs) & $280 / 7-47$ & $221 / 12-391$ & $184 / 20-471$ & 123 & $501 / 620-736$ & 165 \\
\hline Longevity of female (days) & $70 / 112-19$ & $90.0 / 75-243$ & $43.6 / 10-134$ & 72 & $69 / 128-66$ & 48.4 \\
\hline$R_{0} / r_{m} / T^{\mathrm{a}}$ & $-\mid 0.100 /$ & $103.3 / 0.121 / 38.3$ & $92.4 / 0.155 / 29.2$ & $53 / 0.104 / 38$ & $271 / 0.156 / 51.1$ & $50 / 0.160 / 24.4$ \\
\hline Prey consumed during development & $239^{\mathrm{b}}$ & $361 / 325-379^{c}$ & $491 / 303-754^{\mathrm{d}}$ & $184^{\mathrm{c}}$ & - & $1,408^{\mathrm{g}}$ \\
\hline $\begin{array}{l}\text { Prey consumed by ovipositing } \\
\text { females (mites per day) }\end{array}$ & $66.3 / 52-87^{b}$ & $35.9 / 32-44^{e}$ & $46.8 / 11-80^{\mathrm{e}}$ & $67.8^{\mathrm{c}}$ & $294^{\mathrm{f}}$ & $26.1^{g, h}$ \\
\hline
\end{tabular}

${ }^{\text {a }} R_{0}$, net production; $r_{m}$, intrinsic rate of natural increase per day; $T$, mean generation time (days).

b Prey, various instars and adults.

c Prey, proto- and deutonymphs.

d Prey, eggs only.

e Prey, adult females.

f Prey, eggs only, but averaged for only the first 20 days after adult female eclosion.

g Prey, deutonymphs only.

h Adult beetles, males and females, fed.

(2000) found adults of both sexes of $S$. vagans in Australia lived 126 days at $12{ }^{\circ} \mathrm{C}$ compared to only 27 days at $30^{\circ} \mathrm{C}$.

Since the developmental times differ little among species of Stethorini, the differences in $r_{m}$-values between species are attributable to differences in reproductive rates (Mori et al., 2005) (Table 2). At $24-25^{\circ} \mathrm{C}$ the $r_{m}$-values for Stethorus species range from 0.100 in S. punctillum (Roy et al., 2003) to 0.160 in S. loi (Shih et al., 1991); a number of other species have been evaluated and found to fall within this range (Chazeau, 1974a,b; Fiaboe et al., 2007; Mori et al., 2005; Richardson, 1977; Tanigoshi and McMurtry, 1977). Sabelis (1985a,b, 1991) calculates that the $r_{m}$-values for tetranychid mites range from 0.160 to 0.293 at around $25^{\circ} \mathrm{C}$. This is significantly higher than the values for Stethorini species listed in Table 2, but their functional response to prey may still allow them to regulate pest populations.

Few studies have calculated the functional response of Stethorus. Houck (1991) examined handling time as a component of the functional response of $S$. punctum to $T$. urticae, and found that the handling time of starved beetles for each prey increased due to a greater extraction of body fluids from individual prey. Stethorus punctum continued to feed even when satiated with high prey densities and continued to pierce mites, initiate feeding, but then subsequently abandon the prey with minimal extraction of bodily fluids in a classic Type 3 response. This type of response has been observed in other systems as well (Hull et al. 1977a; Haji-Zadeh et al., 1993; Peterson et al., 2000). Types 1 and 2 functional responses have been observed in other Stethorus spp. (Hull et al. 1977a; Gotoh et al., 2004).

\subsubsection{Diapause and overwintering}

All Stethorini in temperate climates overwinter as adults, with a reproductive diapause that is induced by short day lengths and cooler temperatures. Stethorus punctum entered reproductive diapause at day lengths of $10 \mathrm{~h}$ or less and $21-22^{\circ} \mathrm{C}$ (McMurtry et al., 1974). Stethorus japonicus was induced into reproductive diapause with day lengths shorter than $13 \mathrm{~h}$ at $18^{\circ} \mathrm{C}$ (Mori et al., 2005). Species with large geographical ranges that include both temperate and subtropical climates may hibernate in the colder area but not in the warmer (Collyer, 1964; Kaylani, 1967). For example, S. punctum diapauses in Pennsylvania (Colburn and Asquith, 1971; Colburn, 1971) and in Washington state (Horton et al., 2002), but is active year round in southern California
(McMurtry et al., 1970; 1974). Tropical species seldom experience diapause (Chazeau, 1985; Hoy and Smith, 1982).

Several studies have shown that S. punctillum and S. punctum overwinter within fruit orchards and the adjacent habitat. Putman (1955a) and Felland et al. (1995) determined that both species moved into overwintering sites as early as mid-summer (July) and population densities peaked in mid-autumn during apple leaf-fall. In Ontario, Putman (1955a) found that S. punctillum only survived the winter in orchards if they were in contact with the soil, likely because of the insulating effects of snow, whereas dead adults were often observed on the trunks of peaches during the winter. This agrees with observations of S. punctum in Pennsylvania orchards (Hull personal observation). Overwintering Stethorini are often found in aggregations near the base of orchard trees, but this may be a function of increased survival in these protected areas (Asquith and Hull, 1979; Colburn and Asquith, 1971; Felland and Hull, 1996; Readshaw, 1971). Regardless of where they overwinter within a landscape, some North American Stethorus spp. experience substantial mortality during the winter, with spring populations reported as $20-28 \%$ of those found in the fall (Felland and Hull, 1996; Putman, 1955a). In areas where winters are less severe, Stethorus may overwinter successfully in less protected sites. For example, S. punctum commonly overwinter in cardboard bands wrapped around tree trunks in Washington apple orchards (Horton et al., 2002), as does S. punctillum in Germany (Berker, 1958).

Spring emergence of S. punctum adults in Pennsylvania apple orchards over three seasons was correlated with tree phenology, ground cover emergence traps, colored sticky card traps, ambient temperatures, and degree day accumulations (Felland et al., 1995; Biddinger and Hull, 1995). Adults emerged from diapause at 100 and 300 degree days, base $5{ }^{\circ} \mathrm{C}$ starting 1 March of each year,

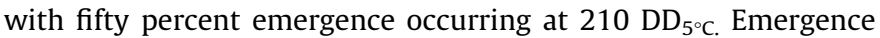
was $2 \%, 47 \%$, and $96 \%$ complete by the defined phenological stages of apple as half inch green, pink and petal fall, respectively, for the apple cultivar "Yorking." Most adults emerged on days of average, minimum, and maximum air temperatures of $15-20,5-15$, and 20-30 ${ }^{\circ} \mathrm{C}$, respectively.

\subsubsection{Natural enemies of Stethorini}

There is surprisingly little information available on the natural enemies of Stethorini species despite numerous reports for other 
coccinellid species (Riddick et al., 2009). A species of rickettsial disease (Rickettsiella stethorae Hall and Badgley) from larvae in cultures of Stethorus nr. punctum from Morocco was described by Hall and Badgley (1957). The disease spread rapidly to cultures of $S$. punctum from Connecticut, S. gilvifrons from Hong Kong, $S$. punctillum, and Stethorus sp., possibly $S$. guatemalensis $=S$. granum introduced from Guatemala (Hall and Fleschner, 1958). The disease was not found in their prey (six-spotted mite, Eotetranychus sexmaculatus [Riley]). Among the parasitic mites, the Laboulbeniales were found on several S. punctillum adults collected on raspberry plants in Québec (Michèle Roy, MAPAQ Laboratoire de Diagnostic en Phytoprotection, pers. comm.). We could find no records of parasitoids that attack Stethorini.

Cannibalism on preimaginal stages of Stethorus is also common (Cottier, 1934; Fleschner, 1950; Robinson, 1953; Chazeau, 1985). It does not appear to be an important cause of mortality except during times of starvation (Collyer, 1953; Putman, 1955a; Kaylani, 1967; Mathur, 1969) or when larval densities become very high (Houck, 1991). Biddinger and Hull (personal observation) believe the most significant predators to be the older instars of lacewings (Chrysopa spp.) and other coccinellids. Phidippus audax (Hentz), a salticid commonly found in the tree canopy in the late summer and fall, feeds on Stethorus larvae (Biddinger and Hull, personal observation). Rosenheim et al. (2004a, b) found the tangle-nest spider, Nesticodes rufipes (Theridiidae), consumed larvae of S. siphonulus, thereby disrupting biological control of the carmine spider mite, Tetranychus cinnabarinus, on papaya in Hawaii. Putman (1955a) observed an unidentified species of Typhlodromus feeding on the eggs of S. punctillum when confined. Haney et al. (1987) found that $S$. punctum could regulate populations of citrus red mite in California citrus groves at low levels, but in the presence of the Argentine ant, Iridomyrmex humilis (Mayr), mite populations flared dramatically. They attributed this to active harassment of adult (and possibly immature stages) Stethorus (also documented by Bartlett, 1963), which inhibited the feeding and numerical response of the beetles.

\section{Conservation and value in biological control}

The habitats of Stethorini are diverse, and include many agricultural systems such as tree and small fruits, nuts, citrus, avocadoes, bananas, papaya, palms, tea, cassava, sugarcane, maize, and various vegetables, as well as ornamental plantings, grasslands, forests, and heathlands. The overview provided by Lo et al. (1990) of tetranychid predators in several crops in Taiwan is an illustration of where acariphagous coccinellids often fit within natural enemy communities. Typically coexisting with several phytoseiid mite predators, the staphylinid Oligota and green lacewings (Chrysopa), Stethorus loi Sasaji is considered an important mite predator in citrus and tea plantations, whereas in mulberry, strawberry, and pear, mite management practices focus completely on phytoseiids. Citrus red mite (Panonychus citri) is preyed upon by a number of Stethorini in Asia, frequently resulting in significant population suppression. For instance, Huang et al. (1988) provide an account of inoculation of approximately 20 adult $S$. siphonulus per tree in six citrus orchards in southeastern China, resulting in reduction of $P$. citri to below economic thresholds over the growing season, and drastic reduction in miticide input.

It is difficult to generalize which agroecosystems support Stethorini and/or phytoseiid mites as effective tetranychid predators; this is likely a function of many factors such as pesticide use history, crop phenology and characteristics, proximity to refugia for protection from climatic extremes and agrochemicals, and experience and preference of pest management practitioners. Globally, Stethorini perform best as biological control agents in low-input woody perennial systems. Key to their optimal utilization as biological control agents are the provision of sufficient overwintering habitats or other reservoirs for Stethorini in or near agroecosystems, and their protection from particularly damaging pesticides. Under these circumstances, Stethorini are often important contributors to the suppression of tetranychid populations. Examples are apple orchards in Pennsylvania, USA, citrus and tea in East and South Asia, and in Australian and New Zealand gorse (Ulex europaeus L. [Fabaceae]), where they suppressed a tetranychid biological control agent for this invasive woody weed.

\subsection{Case studies of Stethorini in IPM systems}

\subsubsection{Stethorus punctum in Pennsylvania orchards}

The best documented and most successful biological control program incorporating Stethorini has been that of S. punctum in apple and peach orchards of the eastern USA. Pennsylvania initiated a system for the biological control of mites using S. punctum during the 1970s (reviewed in Asquith and Hull, 1979; Chazeau, 1985; Croft, 1990; Hull and Beers, 1985; Tanigoshi et al., 1983). The program reportedly reduced acaricide usage by 1000 metric tonnes of formulated product, realizing a cumulative grower savings of US\$20 million over 25 years (Biddinger and Hull, 1995).

Key to the success of this program was early development of resistance by $S$. punctum to organophosphate (OP) insecticides such as azinphosmethyl (Colburn and Asquith, 1973), and the continuous use of this pesticide class in controlling susceptible primary pests (e.g., codling moth and Oriental fruit moth) from the mid 1960s through the mid 1990s (Croft, 1990). The intense selection pressure over decades of using multiple applications of the same insecticides per season undoubtedly contributed to this resistance development, but another factor was a unique method of application known as alternate row-middle spraying (Lewis and Hickey, 1964; Hull and Beers, 1985). In the 1970s this became the preferred method of pesticide application by over $95 \%$ of mid-Atlantic fruit growers. Knight and Hull (1992a,b) demonstrated that, using this method, only $\sim 20 \%$ dose of the pesticide is deposited on leaves or fruit on the opposite side of the tree, leaving untreated refugia for Stethorus, even as new insecticides were employed, to which the predator was not resistant (Hull et al., 1976; Hull and Beers, 1985; David, 1985; Biddinger, 1993; Biddinger and Hull, 1995; Biddinger and Hull, 1999).

The usefulness of Stethorus was communicated directly to tree fruit growers through insecticide and acaricide efficacy guides. A series of field studies and a computer simulation model by Mowery et al. (1975) determined the expected efficacy of biological control of $P$. ulmi based on visual predator and prey counts, information later incorporated into the Penn State Apple Orchard Consultant program, one of the first IPM expert systems (Rajotte et al., 1987; Travis et al., 1992). Pesticide recommendations focused not only on efficacy against primary pests, but on use of products that $S$. punctum could tolerate: OPs and many acaricides (Biddinger et al., 2008a). To protect $S$. punctum, pyrethroids, despite being inexpensive and effective on many primary pests, were never recommended in Pennsylvania apples, and are rarely used there after bloom (Hull and Knight, 1989; Hull and Starner, 1983; Hull et al., 1985a,b). In contrast, in Michigan and New York apples and in Pennsylvania peaches, S. punctum disappeared with widespread adoption of pyrethroids in the late 1980s (Hull, personal observation).

The period of tetranychid mite control in Pennsylvania with $S$. punctum was characterized by a lack of effective miticides. Starting in the mid 1990s, new miticide registrations offered more effective, less expensive materials. Most growers abandoned recommended action thresholds for P. ulmi, and miticide use increased dramatically. Stethorus punctum began to disappear from apple orchards 
as lower populations of $P$. ulmi prevented predator reproduction. Of more lasting impact however, was the development of OP resistance in the primary lepidopterous pests of eastern apple orchards, which required adoption of new insecticide chemistries, some of which were very toxic to $S$. punctum. These insecticides include the neonicotinoids, and several of the insect growth regulators, which are toxic to various stages of S. punctum (Biddinger and Hull, 1993, 1995, 2005; Hull et al., 1991; Hull and Biddinger, 1991a,b), in spite of being classified as "reduced risk," by the US EPA. James (2003b, 2004) reported similar effects on S. punctum picipes in Washington hops. Many of these new insecticides have sublethal effects on pest development and fecundity (Biddinger and Hull, 1999; Sun et al., 2000; Biddinger et al., 2006), which have been demonstrated for $S$. punctillum through feeding on tetranychids on imidacloprid-treated ornamental woody plants (Creary 2009).

Around 2005, biological mite control in Pennsylvania apple orchards shifted to the conservation of the phytoseiid predatory mite, Typhlodromus pyri (Schueten) (Biddinger et al., 2008b). T. pyri can survive on alternative food sources such as rust mites, pollen or fungi when tetranychid mite densities are very low and do not seasonally disperse from trees, as do other phytoseiid predators such as Neoseiulus fallacis (Garman) (Nyrop et al. 1998). Biological mite control with $T$. pyri in Pennsylvania apple orchards does not suffer from temporal or spatial asynchrony such as that found in raspberries (Roy et al. 2005). Stethorus punctum is now considered a backup option for mite control when T. pyri conservation fails due to the use of toxic insecticides rather than a complement to phytoseiids. Currently, neither S. punctum nor T. pyri are providing significant mite control in Pennsylvania peach orchards because of the heavy dependency on pyrethroid applications for pest control (Hull and Biddinger, personal observation).

\subsubsection{Pesticide impacts and resistance in other Stethorini species}

Nienstedt and Miles (2008) have established a bioassay for pesticide toxicity for $S$. punctillum, including effects on development and fecundity, and demonstrated its sensitivity using the insect growth regulators fenoxycarb and methoxyfenozide. This species developed resistance to azinphosmethyl in Italian apple orchards under very similar circumstances to that of $S$. punctum in USA (Pasqualini and Malavolta, 1985; Croft, 1990). The value of this predator has also been reduced as alternative insecticides have been adopted to control azinphosmethyl-resistant primary pests (Pasqualini and Antropoli, 1994). Biological control of mites in Italy is now also more dependent on the phytoseiid predatory mites. $T$. pyri and Amblysieus andersoni (Chant) (Pasqualini, personal communication).

McMurtry et al. (1970) reviewed the biology and ecology of several North American Stethorus spp. and noted the impact of orchard spray practices on them. Stethorus bifidus Kapur was the most important insect predator of mites in New Zealand apple orchards, but is susceptible to organophosphate insecticides (Collyer, 1964, 1976). The use of alternate row-middle applications of reduced rates of insecticides was not adopted in New Zealand orchards or in other countries with native species of Stethorini; this may contribute to the absence of insecticide resistance. The introduction of the synthetic pyrethroids into New Zealand apple spray programs severely impacted Stethorini populations and fruit IPM now largely relies on the introduced pyrethroid resistant phytoseiid, T. pyri (Croft, 1990; Marwick, 1988). In Australian apple IPM programs several species of Stethorini were important in the 1960-1970s, but are now dependent on phytoseiid predators for biological mite control (Edwards and Hodgson, 1973; Readshaw, 1975; Walters, 1974; Walters, 1976a,b,c; Bower and Kaldor, 1980).

Álvarez-Alfageme et al. (2008) examined the effect of two different $\mathrm{Cry} 1 \mathrm{Ab}$ expressing transgenic maize cultivars with lepidoptera-specific Bt toxins. The two-spotted spider mite, T. urticae, retains the Bt toxin but its predator, S. punctillum, degrades it without measureable effects on fitness or performance. This is consistent with field results comparing Cry $1 \mathrm{Ab}$ expressing maize with its isogenic cultivar in Spain, showing no significant differences in coccinellid numbers, which were predominantly $S$. punctillum (de la Poza et al., 2005). Güllü et al. (2004) reported similar results with S. gilvifrons comparing Cry1 AB expressing maize with an isogenic cultivar in Turkey. To our knowledge no specific deleterious findings are available regarding Stethorus and rootworm-targeted (Cry3) transgenic maize.

\subsection{Mass rearing}

Early biological control researchers cavalierly pursued the introduction of many Stethorini into new regions, and accompanying these classical biocontrol introductions, considerable effort went into the development of mass production methods using natural diets of mites and factitious prey or artificial diets. The mass production of Stethorini using prey requires a tremendous supply of mites. Fleschner (1950) conservatively calculated that S. picipes each required 300 mites for development and oviposition. Several species of mites have been used to rear Stethorus including Eotetranychus sexmaculatus (Riley), Tetranychus pacificus McGregor, T. cinnabarinus (Finney, 1953; Scriven and Fleschner, 1960; Scriven and McMurtry, 1971).

Some host plants may not be suitable for cultures of Stethorini because of hooked trichomes that may kill or impede the movement of larvae and adults. For example, prey mites must be brushed from lima or scarlet runner bean plants before offering them to Stethorini as food since the hooked trichomes on these plants can tear the larval integument, and damage the posterior integument during defecation or oviposition by adults (Putman, 1955a; Walters, 1974; Biddinger, 1993). The smooth-leaved fava bean (Vicia faba) proved more suitable for rearing Stethorus directly on the plants (Putman, 1955a; Biddinger, 1993).

Stethorus can also be reared on alternative foods. Colburn (1971) determined that a modified wheat germ diet with honey greatly increased adult $S$. punctum survival in the laboratory over a two week period compared to sugar water alone. Smirnoff (1958) reared S. punctillum and 17 other coccinellids on a diet consisting of cane sugar, honey, agar and royal jelly. Given the limited work done on these non-prey diets, it is difficult to make any firm conclusions as to their value in the mass production of beetles.

Applied Bio-nomics near Victoria, British Columbia, Canada, is the commercial supplier of S. punctillum for releases in the United States and Canada. The company recommends S. punctillum for release against T. urticae, $P$. ulmi, the spruce spider mite Oligonychus ununguis (Jacobi), and the Southern red mite Oligonychus ilicis (McGregor). Since the beetles are expensive (US\$30-50 per 100 adults), they are sold only in modest numbers compared to phytoseiid mite predators, particularly for interior landscapes, conservatories, and greenhouses with ornamental and vegetable crops, as well as for occasional field use on small fruits and ornamental shrubs (Raworth et al., 2002; Jan Dietrich, Rincon-Vitova Insectaries, and Brian Spencer, Applied Bio-nomics Corporation, personal communication, with D. Weber, both on 5 December, 2008).

\subsection{Research on releases in urban, greenhouse, and interior environments}

Several studies have focused on using Stethorini for controlling spider mites in greenhouse crops. Raworth (2001) found that releases of $S$. punctillum established and reproduced in peppers and cucumbers but not in tomatoes. This study underscores yet again that the prey's host plant can inhibit predators. Rott and Ponsonby (2000) found in UK glasshouse vegetables, that simultaneous re- 
leases of Neoseuilus californicus, S. punctillum, and P. persimilis controlled spider mites better than did $P$. persimilis alone; no intraguild predation was noted, and predator performance varied by crop type. Combined releases of phytoseiids and S. punctillum have shown success in greenhouses, interiorscapes, and urban shade tree IPM programs in Europe (Gorski and Fajfer, 2003; Jäckel et al., 2000, 2008; Pöhle et al., 2002). These efforts are testing combinations of releases of phytoseiid mites with $S$. punctillum with habitat modifications, e.g., on shade trees increasing ground residue and providing shelterbands for overwintering. Interior environments with low humidity and artificial lighting pose special challenges that are difficult to overcome (Pöhle et al., 2002). Ornamental pest management is also seen as a possible niche for $S$. punctillum releases in Denmark (Svendsen and Hansen, 2002).

\subsection{Inhibition of gorse biocontrol}

Stethorini may suppress desirable mites that are biological control agents of invasive weeds. Gorse, Ulex europaeus L. (Fabaceae), is a thorny woody shrub native to Europe, which was widely redistributed throughout the world as a living hedge to confine livestock. This plant is also appreciated for its value as browse for live-stock, and for its abundant yellow flowers. By the early 20th century, the dangerously weedy nature of gorse was recognized in New Zealand, southern Australia, Tasmania, Chile, northwestern USA and Hawaii. Beginning about 20 years ago, efforts turned toward classical biological control using two foliage-feeding arthropods, the gorse spider mite, Tetranychus lintearius Dufour (of British and Iberian origin) and gorse thrips, Sericothrips staphylinus Haliday (of English origin). T. lintearius has been released in New Zealand, Australia, Chile, and in the USA (Washington, Oregon, California, and Hawaii).

Established gorse spider mite populations grow to spectacular numbers with webbing that envelops gorse plants. But several regions have seen steep population declines due to predation by Stethorini and phytoseiids. In New Zealand, the native S. bifidus and less commonly the exotic $P$. persimilis suppress gorse spider mite populations to the extent that "after several years populations decline rapidly, and never outbreak again" (Hill et al., 2000). Peterson et al. (2000) found that $S$. bifidis can regulate $T$. lintearius, based on its functional response in laboratory arenas.

In Australia, predation on gorse mite by the native Parastethorus histrio as well as by the exotic phytoseiid $P$. persimilis, was detected soon after initial releases in Tasmania and Victoria, where "it is expected that both predators will significantly restrict its impact" (Ireson et al., 2003, 2004). Davies et al. (2007) estimated that a $36 \%$ reduction in gorse biomass occurred in Tasmanian plots, where both Parastethorus histrio and Phytoseiulus persimilis were common. Without these predators, they estimated a $44 \%$ biomass reduction would have occurred, and this magnitude of effect was considered important in the context of gorse competition with other plants.

In northwestern USA, $P$. persimilis has significantly reduced gorse mite colonies 3-4 years post-establishment (Pratt et al., 2003), and S. punctillum "has also caused severe declines" (Coombs et al., 2004), although the magnitude of the effect on the target weed is uncertain. The situation in Chile was more favorable for gorse mite populations, where, in spite of the presence of $P$. histrio in Chile (Aguilera, 1987), the native staphylinid predator Oligota centralis (Solier) was the only gorse mite predator commonly found, and its impact on T. lintearius was not strong (Norambuena et al., 2007).

Predation on gorse mite populations by Stethorini, which in at least two of the four regions has had a major impact on this classical weed biocontrol effort, should not come as a surprise. In fact, this so-called biotic resistance was observed in Europe by Schroe- der and Zwölfer (1970) in endemic gorse habitats, predicted by Ireson et al. (1999), and continues in the UK, where S. punctillum and phytoseiid predators limit gorse mite outbreaks (e.g., Kirby, 2005).

\section{Conclusions and directions for future work}

Stethorini occur in association with spider mites in a variety of habitats throughout much of the world. Although considered specialist predators, many species function as ecological habitat generalists, able to disperse to multiple plants and to feed on multiple mite species. We have little understanding, however, of these beetles' interaction with their environment other than with their essential prey mites. The clear contributions of Stethorini to population regulation of herbivorous mites in less-disturbed systems (e.g., the disruption of tetranychid-based gorse classical biological control) contrasts with numerous observations that the intense management of cropland exacerbates mite outbreaks by disrupting natural predators. The current and potential roles of Stethorini in biological control can be strengthened by targeting research at several key knowledge gaps.

(1) The role of alternative foods is little known, but may play an important role in population dynamics, diapause, and migration, as with other coccinellids (Lundgren, 2009a,b). Stethorini consume alternative non-tetranychid mite prey, extrafloral nectar and pollen. They are sensitive to plant characteristics and thus cultivar choice and vegetational diversity may increase or hinder their efficacy.

(2) The vision and olfaction of Stethorini are apparently acute, yet their role in prey location remains poorly explored. Research to address the sensory contributions to their impressive dispersal abilities, and to investigate their response to spatially dynamic prey, is likely to be particularly fruitful. Related to this, the regulatory functional responses that appear to prevent tetranychid outbreaks merit further attention.

(3) There is a strong need for systematics research on the group, and for molecular tools to distinguish cryptic species. Stethorini introductions have been common (both accidental and intentional) and careless, and non-target considerations (including the dissemination of entomopathogens and displacement of native species) now dictate a more responsible course. This history of redistribution and the likelihood of significant numbers of yet unnamed species (particularly in Asia) compel the advancement of Stethorini systematics.

(4) Habitat management shows potential for increasing the impact of Stethorini on pest populations. The broad prey and host plant ranges of many species make non-crop habitat valuable sources for predators that can rapidly deploy to colonize and protect crops. These refuges can assist overwintering success in temperate systems, and provide safe havens from pesticides. In milder climates, perennial reservoirs may serve as refuges within diversified annual plantings (e.g., the role of the perennial castor bean, common in Indian farmscapes, which serves as a reservoir for S. gilvifrons and S. pauperculus [Mathur, 1969; Puttaswamy and ChannaBasavanna, 1977]). Use of chemical control, even pesticides that have novel selectivity (e.g., insect growth regulators) or are organic-approved (e.g., elemental sulfur) can be very harmful to Stethorini and other biological agents. These effects are often seen only after registration and widespread use; the breadth of pre-release non-target screening should be broadened (e.g., with method of Nienstedt and Miles, 2008) to include the Stethorini.

The full potential of Stethorini as predators of spider mites will only be realized when cultural and chemical farm management practices are truly integrated with biological controls based on farm-scale scientific experimentation. Where proper conditions have come together, such as in Pennsylvania apple orchards, in 
Asian citrus and tea, and in several other tropical systems, Stethorini play an important role-sometimes the most important rolein suppressing tetranychid populations.

\section{Acknowledgments}

We are grateful to Jon Lundgren and Michèle Roy and to two anonymous reviewers, for helpful comments on an earlier draft. Michael Athanas of IIBBL, Long Zhang of China Agricultural University, Michèle Roy of MAPAQ Laboratoire de Diagnostic en Phytoprotection, and the staff of the National Agricultural Library, located a number of the publications consulted. Meiling Z. Webb of IIBBL, Tina Trenczek of University of Giessen, Germany, Hazem Abdelnabby of Benha University in Egypt, and Hossein Hosseini Moghadam of Gent University in Belgium, provided very useful translations. Mention of any proprietary products does not constitute endorsement by the USDA or the Pennsylvania State University.

\section{References}

Afshari, G.A., 1999. A survey on the ladybirds belonging to the genus Stethorus and study of the biology, prey consumption and population dynamics of Stethorus gilvifrons in sugarcane farms in Khuzestan, Iran. Master Science Thesis, Ahvaz, Iran.

Afshari, M., Mossadegh, S., Soleyman-Nejadian, E., Kamali, K., 2007. Geographica distribution and host plants of Stethorus gilvifrons (Mulsant) (Col.: Coccinellidae) and its biology under laboratory conditions in Khuzestan province. Journal of Agricultural Science and Natural Resources 14, 201-210.

Aguilera, P.A., 1987. Nuevas localidades para Stethorus histrio Chazeau (Coleoptera: Coccinellidae) en Chile. Revista Chilena de Entomologia 15, 33-36.

Ahmed, Z.I., Ahmed, R.F., 1989. Biological studies of the predator Stethorus gilvifrons Mulsant. (Coleoptera: Coccinellidae) on strawberry mites Tetranychus turkestan Ugarov and Nikolski (Acarina: Tetranychidae). Journal of Biological Science Research 20, 22-23.

Al-Duhawi, S.S., Ali, A.A., Sameer, S.H., 2006. The predation efficacy of the predators Stethorus gilvifrons (Muls) and Scolothrips sexmaculatus (Perg.) on tobacco whitefly Bemisia tabaci (Gen) which attack cotton plants. Arabian Journal of Plant Protection 24, 112-117 (English abstract) http:// www asplantprotection.org/PDF/AJPP/24-2_2006/112-117.pdf.

Almatni, W., Khalil, N., 2008. A primary survey of aphid species on almond and peach, and natural enemies of Brachycaudus amygdalinus in As-Sweida, Southern Syria. In: Boos, M. (Ed.), Ecofruit-13th International Conference on Cultivation Technique and Phytopathological Problems in Organic FruitGrowing: Proceedings to the Conference from 18-20 February 2008, Weinsberg, Germany, pp. 109-115. http://orgprints.org/13654/.

Álvarez-Alfageme, F. Ferry, N., Castanera, P., Ortego, F., Gatehouse, A.M.R., 2008. Prey mediated effects of Bt maize on fitness and digestive physiology of the red spider mite predator Stethorus punctillum Wiese (Coleoptera: Coccinellidae) Transgenic Research 17, 943-954.

Ansari, M.A., Pawar, A.D., 1992. Biology of spider mite, Tetranychus ludeni Zacher (Acari: Tetranychidae) recorded on waterhyacinth. Plant Protection Bulletin (Faridabad) 44, 28-31.

Asquith, D., Hull, L.A., 1979. Integrated pest management system in Pennsylvania apple orchards. In: Boethel, D.J., Eikenbary, R.D. (Eds.), Pest Management Programs for Deciduous Tree Fruits and Nuts. Plenum Press, New York, pp. 203220.

Bailey, P., Caon, G., 1986. Predation of twospotted mite, Tetranychus urticae Koch (Acarina: Tetranychidae), by Halothrips victoriensis Bagnall (Thysanoptera: Phlaeothripidae) and Stethorus nigripes Kapur (Coleoptera: Coccinellidae) on seed lucerne crops in South Australia. Australian Journal of Zoology 34, 515525.

Bartlett, B.R., 1963. The contact activity of some pesticide residues to hymenopterous and coccinellid predators. Journal of Economic Entomology 56, 694-698.

Beattie, G.A.C., Gellatley, J.G., 1983. Mite pests of citrus. Agfacts (Australia), Publication H2.AE.3, 6 pp.

Berker, J., 1958. Die natürlichen Feinde der Tetranychiden. Zeitschrift für Angewandte Entomologie 43, 115-172.

Biddinger, D.J., 1993. Toxicity, stage specificity, and sublethal effects of abamectin and several classes of insect growth regulators to Platynota idaeusalis (Lepidoptera: Tortricidae) and Stethorus punctum (Coleoptera: Coccinellidae). Ph.D. dissertation, The Pennsylvania State University, 199 pp.

Biddinger, D.J., Hull, L.A., 1993. Apple, Effects of insect growth regulators on Stethorus punctum, 1992. Insecticide and Acaricide Tests 18, 3-4.

Biddinger, D.J., Hull, L.A., 1995. Effects of several types of insecticides on the mite predator, Stethorus punctum (Coleoptera: Coccinellidae), including insect growth regulators and abamectin. Journal of Economic Entomology 88, 358366.
Biddinger, D.J., Hull, L.A., 1999. Sublethal effects of selected insecticides on growth and reproduction of a laboratory susceptible strain of tufted apple bud moth (Lepidoptera: Tortricidae). Journal of Economic Entomology 92, 314-324.

Biddinger, D.J., Hull, L.A., 2005. Survey of Pennsylvania apple orchards for a mite predator to give effective and sustainable control of spider mites. Penn Fruit News 85, 23-28.

Biddinger, D.J., Huang, H., Hull, L.A., McPheron, B.A., Loyer, M., 2006. Sublethal effects of chronic exposure to tebufenozide on development, survival, and reproduction of the tufted apple bud moth (Lepidoptera: Tortricidae). Journal of Economic Entomology 99, 834-842.

Biddinger, D., Hull, L., Krawczyk, G., 2008a. Biological control of mites. In: R. Crassweller, R. (Ed.), Pennsylvania Tree Fruit Production Guide 2008-2009 edition. Penn State Cooperative Extension. AGRS-45, pp. 123-128, 195. http:// tfpg.cas.psu.edu/.

Biddinger, D., Hull, L., Krawczyk, G., 2008b. Final Report to the Pennsylvania Department of Agriculture Project \# ME 444201, 2005-7. Biological mite control in Pennsylvania apple orchards through the distribution and conservation of the predatory mite, Typhlodromus pyri. $13 \mathrm{pp}$. http://resources.cas.psu.edu/ipm/ nrcs/amabcspec07.pdf.

Bower, C.C., Kaldor, J., 1980. Selectivity of five insecticides for codling moth control: effects on the twospotted spider mite and its predators. Environmental Entomology 9, 128-132.

Britto, E.P.J., Gondim Jr., M.G.C., Torres, J.B., Fiaboe, K.K.M., Moraes, G.J, Knapp, M., 2009. Predation and reproductive output of the ladybird beetle Stethorus tridens preying on tomato red spider mite Tetranychus evansi. BioControl. doi:10.1007/ s10526-008-9178-5.

Butcher, F.G., 1951. Some observations on various insects found on fruit and ornamentals in the Miami area. Proceedings of the Florida State Horticultural Society 1951, 251-254.

Chang, D.C., Leu, T.S., 1986. Seasonal population changes of spider mites on carambola and their chemical control. Plant Protection Bulletin of Taiwan 28, 263-272.

Charles, J.G., Collyer, E., White, V., 1985. Integrated control of Tetranychus urticae with Phytoseiulus perimilis and Stethorus bifidus in commercial raspberry gardens. New Zealand Journal of Experimental Agriculture 13, 385-393.

Chazeau, J., 1971a. Le genre Stethorus à Madagascar et aux Mascareignes (Col.: Coccinellidae). Annales de la Société Entomologique de France (N.S.) 7, 779796

Chazeau, J., 1971b. Stethorus comoriensis, nouvelle espèce de Coccinellidae de l'archipel des Comores (Col.). Bulletin de la Société Entomologique de France 76, 60-63.

Chazeau, J., 1974a. Développement et fécondité de Stethorus madecassus Chazeau (Coléoptères, Coccinellidae) élevé en conditions extérieures dans le sud-ouest de Madagascar. Cahiers ORSTOM, Série Biologie 25, 27-33.

Chazeau, J., 1974b. Evaluation de l'action prédatrice de Stethorus madecassus (Coléoptères, Coccinellidae) sur Tetranychus neocaledonicus (Acarien Tetranychidae). Entomophaga 19, 183-193.

Chazeau, J., 1979. Mise au point sur le genre Stethorus en Océanie et description de deux espèces nouvelles de Mélanésie (Col. Coccinellidae). Entomophaga 24, 295-303.

Chazeau, J., 1983. Deux prédateurs de Tetranychidae en Nouvelle-Guinée: Stethorus exspectatus n.sp. et Stethorus exsultabilis n.sp. (Col.: Coccinellidae). Entomophaga 28, 373-378.

Chazeau, J., 1985. Predaceous insects. In: Helle, W., Sabelis, M.W. (Eds.), Spider Mites; Their Biology, Natural Enemies, and Control, vol. B. Elsevier, Amsterdam, pp. 211-246.

Chazeau, J., Etienne, J., Fürsch, H., 1974. Les Coccinellidae de l'íle de La Réunion (Insecta Coleoptera). Bulletin du Museum d'Histoire Naturelle, Zoologie 210, 265-297.

Chen, W., 1993. Predation behavior of Stethorus chengi Sasaji on patchily distributed preys. Journal of Shanghai Agricultural College (China) 11, 209-213 (in Chinese).

Chen, W., Zhao, Z., 1994. Study on predation of Stethorus chengi Sasaji to Panonychus citri McGregor. Journal of the Southwest Agricultural University 16, 27-31 (in Chinese).

Chen, Y., Wei, S., Zhang, L., 2005. Status of banana R\&D in Hainan, China. In: Molina, A.B., Xu, L.B., Roa, V.N., Van den Bergh, I., Borromeo, K.H. (Eds.), Advancing Banana and Plantain R\&D in Asia and the Pacific. Proceedings of the 3rd BAPNET Steering Committee meeting held in Guangzhou, China, 23-26 November 2004, vol. 13. International Network for the Improvement of Banana and Plantain, Montpellier, France, pp. 193-201.

Cinti, S., Storti, C., Vitagliano, A., Nanelli, R., Paparatti, B., 1995. Prime osservazioni sulla biologia di Oligonychus bicolor (Banks) (Acari, Tetranychidae) nel comprensorio castanicolo dei Monti Cimini. Informatore Fitopatologico 45 (3), 60-62.

Colburn, R.B., 1971. The predator Stethorus punctum (Coleoptera: Coccinellidae), and its relationship to Panonychus ulmi (Acarina: Tetranychidae). Ph.D. Thesis, Pennsylvania State University.

Colburn, R.B., Asquith, D., 1970. A cage used to study the finding of a host by the ladybird beetle, Stethorus punctum. Journal of Economic Entomology 63, 13761377.

Colburn, R.B., Asquith, D., 1971. Observations on the morphology and biology of the ladybird beetle Stethorus punctum. Annals of the Entomological Society of America 64, 1217-1221.

Colburn, R.B., Asquith, D., 1973. Tolerance of Stethorus punctum to adults and larvae to various pesticides. Journal of Economic Entomology 66, 961-962. 
Collyer, E., 1953. Biology of some predatory insects and mites associated with the fruit tree spider mite Metatetranychus ulmi (Koch) in south-eastern England. II. Some important predators of the mite. Journal of Horticultural Science 27, 8597.

Collyer, E., 1964. Phytophagous mites and their predators in New Zealand orchards. New Zealand Journal of Agricultural Research 7, 551-568.

Collyer, E., 1976. Integrated control of apple pests in New Zealand. 6. Incidence of European red mite, Panonychus ulmi (Koch), and its predators. New Zealand Journal of Zoology 3, 39-50.

Congdon, B.D., Shanks Jr., C.H., Antonelli, A.L., 1993. Population interactions between Stethorus punctum picipes (Coleoptera: Coccinellidae) and Tetranychus urticae (Acari: Tetranychidae) in red raspberries at low predator and prey densities. Environmental Entomology 22, 1302-1307.

Coombs, E., Clark, J., Piper, G., Cofrancesco Jr., A., 2004. Biological Control of Invasive Plants in the United States. Oregon State University Press, Corvallis.

Cottier, W., 1934. The natural enemies of the European red mite in New Zealand. New Zealand Journal of Science and Technology 16, 68-80.

Creary, S., 2009. Indirect effects of imidacloprid on two predators of spider mite on elms and boxwoods. Master of Science Thesis, Department of Entomology, University of Maryland, College Park.

Croft, B.A., 1990. Arthropod Biological Control Agents and Pesticides. Wiley, New York.

David, P.J., 1985. Ovicidal activity of methomyl on eggs of pest and beneficial insects and the mites associated with apples in Virginia. Journal of Economic Entomology 82, 432-436.

Davies, J.T., Ireson, J.E., Allen, G.R., 2007. The impact of the gorse spider mite, Tetranychus lintearius, on the growth and development of gorse, Ulex europaeus. Biological Control 41, 86-93.

Dean, H.A., 1957. Predators of Oligonychus pratensis (Banks), Tetranychidae. Annals of the Entomological Society of America 50, 164-165.

de la Poza, M., Pons, X., Farinósa, G.P., López, C., Ortego, F., Eizaguirre, M., Castañera, P., Albajes, R., 2005. Impact of farm-scale Bt maize on abundance of predatory arthropods in Spain. Crop Protection 24, 677-684.

Dhooria, M.S., 1981. Feeding behavior of predatory mites, thrips and beetles of the citrus mite Eutetranychus orientalis. Acarology Newsletter 10, 4-6.

Edwards, B.A.B., Hodgson, P.J., 1973. The toxicity of commonly used orchard chemicals to Stethorus nigripes (Coleoptera: Coccinellidae). Journal of the Australian Entomological Society 12, 222-224.

Ehler, L.E., 1974. A review of the spider-mite problem on grain sorghum and corn in West Texas. Texas Agricultural Experiment Station Bulletin 1149, 15 pp.

Evans, E.W., 2009. Lady beetles as predators of insects other than Hemiptera. Biological Control 51, 255-267.

Evans, J.W., 1943. Insect Pests and Their Control. Department of Agriculture, Tasmania, Australia.

Felland, C.M., Hull, L.A., 1996. Overwintering of Stethorus punctum punctum (Coleoptera: Coccinellidae) in apple orchards ground cover. Environmental Entomology 25, 972-976.

Felland, C.M., Biddinger, D.J., Hull, L.A., 1995. Overwintering emergence and trapping of adult Stethorus punctum punctum (Coleoptera: Coccinellidae) in Pennsylvania apple orchards. Environmental Entomology 24, 110-115.

Fiaboe, K.K.M., Gondim, M.G.C., de Moraes, G.J., Ogol, C.K.P.O., Knapp, M., 2007. Bionomics of the acarophagous ladybird beetle Stethorus tridens fed Tetranychus evansi. Journal of Applied Entomology 131, 355-361.

Field, R.P., 1979. Integrated pest control in Victorian peach orchards: the role of Stethorus spp. (Coleoptera: Coccinellidae). Journal of the Australian Entomological Society 18, 315-322.

Finney, G.L., 1953. A technique for mass-culture of six-spotted spider mite. Journal of Economic Entomology 46, 712-713.

Fleschner, C.A., 1950. Studies on searching capacity on the larvae of three predators of the citrus red mite (Paratetranychus citri) (Stethorus picipes, Conwentzia hageni, Chrysopa claifornicus). Hilgardia 20, 233-265.

Fournier, V., Rosenheim, J.A., Johnson, M.W., Brodeur, J., 2002. Augmentative releases of predatory mites on papaya in Hawaii: failures and success. In: $1 \mathrm{st}$ International Symposium on Biological Control of Arthropods Honolulu, Hawaii, USA, January 14-18, 2002.

Gillespie, D.R., Quiring, D.J.M., Greenwood, M., 1997. Collection and selection of natural enemies of twospotted spider mites for biological control. Journal of the Entomological Society of British Columbia 94, 7-11.

Giorgi, J.A., Vandenberg, N.J., McHugh, J.V., Forrester, J.A., Ślipiński, S.A., Miller, K.B., Shapiro, L.R., Whiting, M.F., 2009. The evolution of food preferences in Coccinellidae. Biological Control 51, 215-231.

Gordon, R.D., 1982. New species and new synonymy in neotropical Stethorus Weise (Coleoptera: Coccinellidae). The Coleopterists Bulletin $36,121-126$.

Gordon, R.D., 1985. The Coccinellidae (Coleoptera) of America North of Mexico. Journal of the New York Entomological Society 93, 88-99.

Gordon, R.D., 1993. Stethorus nigripes Kapur new to North America, and a new synonym in Stethorus Weise (Coleoptera: Coccinellidae). Southwestern Entomologist 18, 67-68.

Gordon, R.D., Anderson, D.M., 1979. The genus Stethorus Weise (Coleoptera: Coccinellidae) in Chile. The Coleopterists Bulletin 33, 121-126.

Gordon, R.D., Chapin, E.A., 1983. A revision of the new world species of Stethorus Weise (Coleoptera: Coccinellidae). Transactions of the American Entomological Society (Philadelphia) 109, 229-276.

Gorski, R., Fajfer, B., 2003. Control of red spider mite on indoor crops using the ladybird Stethorus punctillum. Ochrona Roslin 47, 10-11 (in Polish).
Gotoh, T., Nozawa, M., Yamaguchi, K., 2004. Prey consumption and functional response of three acarophagous species to eggs of the two-spotted spider mite in the laboratory. Applied Entomology and Zoology 39, 97-105.

Guanilo, D.A., Martínez, N., 2007. Predadores asociados a Panonychus citri McGregor (Acari: Tetranychidae) en la costa central del Perú. Ecología Aplicada 6, 119129.

Güllü, M., Tatli, F., Kanat, A.D., İslamoğlu, M., 2004. Population development of some predatory insects on Bt and non-Bt maize hybrids in Turkey. International Organization for Biological Control Western Palearctic Regional Section Bulletin 27, 85-91.

Gupta, Y.N., 2001. A conspectus of natural enemies of phytophagous mites and mites as potential biocontrol agents of agricultural pests in India. In: Halliday, R., Walter, D., Proctor, H., Norton, R., Colloff, M. (Eds.), International Congress of Acarology, 10th, Collingwood, Australia. CSIRO Publishing, pp. 484-497.

Haji-Zadeh, J., Kamali, G.K., Assadi, H.B., 1993. Investigations on the functional response and populations fluctuations of Stethorus gilvifrons on red spider mite, Panonychus ulmi (Koch) in Karaj vicinity [Iran]. Applied Entomology and Phytopathology 61, 32-34 (in Farsi).

Hall, I.M., Badgley, M.E., 1957. A Rickettsial disease of larvae of species of Stethorus caused by Rickettsiella stethorae, N. sp.. Journal of Bacteriology 74, 452455.

Hall, J.C., Fleschner, C.A., 1958. A new species of Stethorus Weise from Guatemala now being released in California. The Pan-Pacific Entomologist 34, 98-100.

Handoko, 2004. Preference of Stethorus gilvifrons Mulsant (Coleoptera: Coccinellidae) for spider mites infesting orange (Citrus spp.), apple (Malus spp.) and rose (Rosa spp.) plants. M.S. dissertation, Philippines University of Los Baños, College, Laguna (Philippines), 64 pp.

Haney, P.B., Luck, R.F., Moreno, S.S., 1987. Increases in densities of the citrus red mite, Panonychus citri (Acarina: Tetranychidae) in association with the argentine ant, Iridomyrmex humilis (Hymenoptera: Formicidae), in southern California citrus. Entomophaga 32, 49-57.

Helle, W., Sabelis, M.W. (Eds.), 1985. Spider Mites; Their Biology, Natural Enemies, and Control, vols. A and B. Elsevier, Amsterdam.

Hill, R.L., Gourlay, A.H., Fowler, S.V., 2000. The biological control program against gorse in New Zealand. In: Spencer, N.R. (Ed.), Proceedings of the Tenth International Symposium on Biological Control of Weeds, 4-14 July 1999, Montana State University, Bozeman, Montana, USA, pp. 909-917.

Hodek, I., Honěk, A., 1996. Ecology of Coccinellidae. Kluwer Academic, Dordrecht.

Hodek, I., Honěk, A., 2009. Scale insects, mealybugs, whiteflies and psyllids (Hemiptera, Sternorrhyncha) as prey of ladybirds. Biological Control 51, 232 243.

Horton, D.R., Broers, D.A., Hinojosa, T., Lewis, T.M., Miliczky, E.R., Lewis, R.R., 2002 Diversity and phenology of predatory arthropods overwintering in cardboard bands placed in pear and apple orchards of central Washington State. Annals of the Entomological Society of America. 95, 468-480.

Houck, M.A., 1985. Predatory behavior of Stethorus punctum (Coleoptera: Coccinellidae) in response to the prey Panonychus ulmi and Tetranychus urticae (Acarina: Tetranychidae). Ph.D. Thesis, The Pennsylvania State University, $154 \mathrm{pp}$.

Houck, M.A., 1986. Prey preference in Stethorus punctum (Coleoptera: Coccinellidae). Environmental Entomology 15, 967-970.

Houck, M.A., 1991. Time and resource partitioning in Stethorus punctum (Coleoptera: Coccinellidae). Environmental Entomology 20, 494-497.

Houck, M.A., Strauss, R.E., 1985. The comparative study of functional responses: experimental design and statistical interpretation. The Canadian Entomologist $117,617-629$.

Houston, K.J., 1980. A revision of the Australian species of Stethorus Weise (Coleoptera: Coccinellidae). Journal of the Australian Entomological Society 19, 81-91.

Hoy, M.A., Smith, K.B., 1982. Evaluation of Stethorus nigripes (Col.: Coccinellidae) for biological control of spider mites in California almond orchards. Entomophaga 27, 301-310.

Huang, P.K., Luo, X.N., Song, S.L., 1988. Biological control of Panonychus citri by releasing Stethorus siphonulus Kapur (Coleoptera: Coccinellidae) in southeastern China. Acta Phytophylactica Sinica 15, 1-6 (in Chinese)

Huffaker, C.B., van de Vrie, M., McMurtry, J.A., 1970. Ecology of tetranychid mites and their natural enemies: a review. II. Tetranychid populations and their possible control by predators: an evaluation. Hilgardia 40, 391-458.

Hull, L.A., Beers, E.H., 1985. Ecological selectivity: modifying chemical control practices to preserve natural enemies. In: Hoy, M.A., Herzog, D.C. (Eds.), Biological Control in Apple IPM Systems. Academic Press Inc., New York., pp. 103-122.

Hull, L.A., Biddinger, D.J., 1991a. Apple, effects of insect growth regulators on Stethorus punctum egg viability. Insecticide and Acaricide Tests 16, 14

Hull, L.A., Biddinger, D.J., 1991b. Apple, evaluation of RH-5992 on Stethorus punctum, 1990. Insecticide and Acaricide Tests. 16, 14-15.

Hull, L.A., Knight, A.L., 1989. Effect of late-season fenvalerate and flucythrinate applications on European red mite (Acari: Tetranychidae) and tufted apple bud moth (Lepidoptera: Tortricidae) populations in apple. Journal of Economic Entomology 82, 1174-1179.

Hull, L.A., Starner, V.R., 1983. Impact of four synthetic pyrethroids on major natural enemies and pests of apple in Pennsylvania. Journal of Economic Entomology $76,122-130$.

Hull, L.A., Asquith, D., Mowery, P.D., 1976. Distribution of Stethorus punctum in relation to densities of the European red mite. Environmental Entomology 5 337-342. 
Hull, L.A., Asquith, D., Mowery, P.D., 1977a. The functional responses of Stethorus punctum in relation to densities of the European red mite. Environmental Entomology 6, 85-90.

Hull, L.A., Asquith, D., Mowery, P.D., 1977b. The mite searching ability of Stethorus punctum within an apple orchard. Environmental Entomology 6, 684-688.

Hull, L.A., Barrett, B.A., Rajotte, E.G., 1991. Foliar persistence and effect of fenoxycarb on Platynota idaeusalis (Lepidoptera: Tortricidae) on apple. Journal of Economic Entomology 84, 965-970.

Hull, L.A., Beers, E.H., Meagher Jr., R.L., 1985a. Impact of pesticides on natural enemies in large-orchard trials. Journal of Economic Entomology 78, 163-168.

Hull, L.A., Beers, E.H., Meagher Jr., R.L., 1985b. Integration of biological and chemical control tactics for apple pests through selective timing and choice of synthetic pyrethroid insecticides. Journal of Economic Entomology 78, 714-721.

Idder, M.A., Pintureau, B., 2008. Efficacité de la coccinelle Stethorus punctillum (Weise) comme predateur de l'acarien Oligonychus afrasiaticus (McGregor) dans les palmeraies de la region d'Ouargla en Algerie. Fruits (Paris) 63, 85-92.

Ireson, J.E., Gourlay, A.H., Kwong, R.M., Holloway, R.J., Chatterton, W.S., 1999. Progress on the rearing, release and establishment of the gorse spider mite Tetranychus lintearius Dufour, for the biological control of gorse in Australia. In Bishop, A.C., Boersma, M., Barnes, C.D. (Eds.), Proceedings of the 12th Australian Weeds Conference, 12-16 September 1999, Hobart, Tasmania. Tasmanian Weed Society, Devonport, pp. 320-324.

Ireson, J.E., Gourlay, A.H., Kwong, R.M., Holloway, R.J., Chatterton, W.S., 2003. Host specificity, release, and establishment of the gorse spider mite, Tetranychus lintearius Dufour (Acarina: Tetranychidae) for the biological control of gorse, Ulex europaeus L. (Fabaceae) in Australia. Biological Control 26, 117-127.

Ireson, J.E., Kwong, R.M., Gourlay, H., Davies, J.T., Holloway, R.J., Chatterton, W.S., 2004. Progress on the biological control of gorse (Ulex europaeus) in Australia In: Cullen, J.M., Briese, D.T., Kriticos, D.J., Lonsdale, W.M., Morin, L., Scott, J.K. (Eds.), Proceedings of the XI International Symposium on Biological Control of Weeds, Canberra, Australia, 27 April 2 May, 2003. CSIRO Entomology, Canberra, Australia, pp. 415-418.

Jäckel, B., Balder, H., Schneider, K. Pradel, B, 2000. Stethorus punctillum, an important antagonist of spidermites in the city (abstract). Mitteilungen aus de Biologischen Bundesanstalt für Land- und Forstwirtshaft 370, 291.

Jäckel, B., Balder, H., Hasselmann, K., 2008. Möglichkeiten zur Förderung und zum Einsatz von Nütlingen an Stadtbäumen. Pflanzenschutzamt Berlin, Internal report.

James, D.G., 2003a. Synthetic herbivore-induced plant volatiles as field attractants for beneficial insects. Environmental Entomology 32, 977-982.

James, D.G., 2003b. Pesticide susceptibility of two coccinellids (Stethorus punctum picipes and Harmonia axyridis) important of biological control of mites and aphids in Washington hops. Biocontrol Science and Technology 13, 253-259.

James, D.G., 2004. Effect of buprofezin on survival of immature stages of Harmonia axyridis, Stethorus punctum picipes (Coleoptera: Coccinellidae), Orius tristicolor (Hemiptera: Anthocoridae), and Geocoris spp. (Hemiptera: Geocoridae). Journal of Economic Entomology 97, 900-904.

James, D.G., 2005. Further field evaluation of synthetic herbivore-induced plant volatiles as attractants for beneficial insects. Journal of Chemical Ecology 31 481-495.

James, D.G., Price, T.S., 2004. Field testing of methyl salicylate for recruitment and retention of beneficial insects in grapes and hops. Journal of Chemical Ecology $30,1613-1628$

Kapur, A.P., 1948. On the Old World species of the genus Stethorus Weise (Coleoptera, Coccinellidae). Bulletin of Entomological Research 39, 297-320.

Kapur, A.P., 1950. A new species of Stethorus Weise from India (Coleoptera: Coccinellidae). Proceedings of the Royal Entomological Society of London 19, 148-149.

Kaylani, S., 1967. Biology and life-history of Stethorus gilvifrons Mulsant in Lebanon. Magon Publication Serie Scientifique (Beirut) 11, 1-24.

Khan, I., Spooner-Hart, R., Bailey, P., Khalil, S.K., Ullah, F., 2002. Morphologica studies of the predatory ladybird beetle Stethorus vagans (Blackburn) (Coleoptera: Coccinellidae). Journal of Asia-Pacific Entomology 5, 29-33.

Kirby, M., 2005. Gorse mites and their predators. British Wildlife 16, 314-317.

Kishimoto, H., Adachi, I., 2008. Predation and oviposition by predatory Stethorus japonicaus, Oligota kashmirica benefica, and Scolothrips takahashii in egg patches of various spider mite species. Entomologia Experimentalis et Applicata 128, 294-302.

Knight, A.L., Hull, L.A., 1992a. Linking insecticide bioassays with residue analysis to evaluate control of Platynota idaeusalis (Lepidoptera: Tortricidae) neonates on apple: Seasonal spray program. Journal of Economic Entomology 85, 926-931.

Knight, A.L., Hull, L.A., 1992b. Linking insecticide bioassays with residue analysis to evaluate control of Platynota idaeusalis (Lepidoptera: Tortricidae) neonates on apple: single spray. Journal of Economic Entomology 85, 932-938.

Laffi, F., 1982. Il ragnetto giallo della vite Eotetranychus carpini Oud. f. vitis Dosse. Informatore Fitopatologico 32 (6), 31-34

Lentz, C., Petersen, G., Mölck, G., Wyss, U., 2004. Olfaktorische Orienterung des Spinnmilbenräubers Stethorus punctillum. Mitteilungen der Deutschen Gesellschaft für allgemeine und angewandte Entomologie 14, 191-194.

Lewis, F.H., Hickey, K.D., 1964. Pesticide application from one side on deciduous fruit trees. Pennsylvania Fruit News 43, 13-24.

Li, W.Q., Yang, H.H., Deng, G.R., Jin, M.X., 1990. Study on bionomics of Stethorus guangxiensis and S. Aptus. Journal of Guangxi Agricultural College 9 (4), 19-25 (in Chinese)

Lin, Y.M., Chen, P.Z., 1984. Preliminary observations on Stethorus (Allostethorus) parapauperculus. Natural Enemies of Insects 6, 126-128 (in Chinese).
Lo, K.C., Lee, W.T., Wu, T.K., Ho, C.C., 1990. Use of predators for controlling spider mites (Acarina, Tetranychidae) in Taiwan, China. FFTC NARC International Seminar on the use of parasitoids and predators to control agricultural pests. Tukuba Science City, Ibaraki-ken 305 Japan, October 2-7, 1989. Tukuba-gun, Japan, National Agricultural Research Centre (NARC), 20 pp.

Lorenzato, D., 1987. Controle biologico de acaros fitofagos na cultura da macieira no municipio de Farroupilha, RS. Agronomia-Sulriograndense 23, 167-183.

Lui, Z.G., Lui, N.Z., 1986. A preliminary report on Tetranychus piercei McGregor. Insect Knowledge 23, 18-19 (in Chinese).

Lundgren, J.G., 2009a. Relationships of Natural Enemies and Non-prey Foods. Springer, Dordrecht, The Netherlands.

Lundgren, J., 2009b. Nutritional aspects of non-prey foods in the life histories of entomophagous Coccinellidae. Biological Control 51, 294-305.

Marwick, N.P., 1988. Evaluation of field- and laboratory-selected pyrethroid resistant predatory mites. In: Proceedings of the 18th International Congress of Entomology, Vancouver, British Columbia, Canada, p. 316.

Massee, A.M., 1940. Notes on some interesting insects observed Annual Report [1939]. East Malling Research Station 27, 70-73.

Mathur, L.M.L., 1969. Bionomics of Stethorus gilvifrons Mulsant (Coleoptera: Coccinellidae). The Madras Agricultural Journal 56, 7-11.

McMurtry, J.A., Croft, B.A., 1997. Life-styles of phytoseiid mites and their roles in biological control. Annual Review of Entomology 42, 291-321.

McMurtry, M.A., Johnson, H.G., 1966. An ecological study of the spider mite Oligonychus punicae (Hirst) and its natural enemies. Hilgardia 37, 363402.

McMurtry, J.A., Johnson, H.G., Scriven, G.T., 1969. Experiments to determine effects of mass release of Stethorus picipes on the level of infestations of the avacado brown mite. Journal of Economic Entomology 62, 1216-1221.

McMurtry, J.A., Huffaker, C.B., Van de Vrie, M., 1970. Ecology of tetranychid mites and their natural enemies: a review. I. Tetranychid enemies: their biological characters and the impact of spray practices. Hilgardia 40, 331-390.

McMurty, M.A., Scriven, G.T., Malone, R.S., 1974. Factors affecting the oviposition of Stethorus picipes (Coleoptera: Coccinellidae), with special reference to photoperiod. Environmental Entomology 3, 123-127.

Mehr-Khou, F., Fathipour, Y., Talebi, A.A., Kamali, K., Naseri, B., 2008. Population density and spatial distribution patterns of Tetranychus urticae (Acari, Tetranychidae) and its predator Stethorus gilvifrons (Coleoptera: Coccinellidae) on different agricultural crops. Journal of the Entomological Research Society (Ankara) 10, 23-36.

Mora Morin, J., 1991. Stethorus utilis (Coleoptera: Coccinellidae) pequeña cotorrita que se alimenta de acaros tetraniquidos que atacan a los citricos. Comunicacion corta. Centro Agrícola (Cuba) 18, 83-84

Mori, K., Nozawa, M., Arai, K., Gotoh, T., 2005. Life-history traits of the acaraphagous lady beetle, Stethorus japonicus at three constant temperatures. BioControl 50 35-51.

Mouitia, L.A., 1958. Contribution to the study of some phytophagous mites and their predators in Mauritius. Bulletin of Entomological Research 49, 59-75.

Mowery, P.D., Asquith, D., Bode, W.M., 1975. Computer simulation for predicting the number of Stethorus punctum needed to control the European red mite in Pennsylvania apple trees. Journal of Economic Entomology 68, 250-254.

Muma, M.H., 1955. Lady beetles found on citrus in Florida. Florida Entomolgist 38, 117-124.

Nienstedt, K.M., Miles, M., 2008. Aged-residue method for evaluating toxicity of plant protection products to Stethorus punctillum (Weise) (Coleoptera: Coccinellidae). International Organization for Biological Control Western Palearctic Regional Section Bulletin 35, 122-127.

Norambuena, H., Martinez, G., Carrillo, R., Neira, M., 2007. Host specificity and establishment of Tetranychus lintearius (Acari: Tetranychidae) for biological control of gorse, Ulex europaeus (Fabaceae) in Chile. Biological Control 40, 204212 .

Nyrop, J.P., English-Loeb, G., Roda, A., 1998. Conservation biological control of spider mites in perennial cropping systems. In: Barbosa, P. (Ed.), Conservation Biological Control. Academic Pres, San Diego, California, USA, pp. 307-333.

Obrycki, J.J., Harwood, J.D., Kring, T.J., O’Neil, R.J., 2009. Aphidophagy by Coccinellidae: application of biological control in agroecosystems. Biological Control 51, 244-254.

Paschoal, A.D., 1970. Contribuicao ao conhecimento da familia Tetranychidae no Brasil (Aracnida: Acarina). Thesis, Escola superior de Agriculturea, Universidade de Sao Paulo, Brasil, 116 pp.

Pasqualini, E., Antropoli, A., 1994. Stethorus punctillum. Informatore Fitopatologico 44 (5), 33-36 (in Italian).

Pasqualini, E., Malavolta, C., 1985. Possibilità di contenimento naturale di Panonychus ulmi (Koch) (Acarina: Tetranychidae) su melo in Emilia-Romagna. Bollettino del l'Istituto di Entomologia del l'Universita degli Studi di Bologna 39, 221-230.

Pemberton, R.W., Vandenberg, N.J., 1993. Extrafloral nectar feeding by ladybird beetles (Coleoptera: Coccinellidae). Proceedings of the Entomological Society of Washington 95, 139-151.

Perez, R.P., Almaguel, L., Caceres, I., Feito, E., de la Torre, E., 2004. Los depredadores de Tetranychus tumidus Banks en Cuba. Fitosanidad 8, 47-50.

Peterson, P.G., McGregor, P.G., Springett, B.P., 1994. Development of Stethorus bifidus in relation to temperature: implications for regulation of gorse mite populations. In: Popay, E.J. (Ed.), Proceedings of the 47th New Zealand Plant Protection Conference. New Zealand Weed and Pest Control Society Inc., Palmerston North, New Zealand, pp. 103-106. 
Peterson, P.G., McGregor, P.G., Springett, B.P., 2000. Density dependent preyfeeding time of Stethorus bifidus (Coleoptera: Coccinellidae) on Tetranychus lintearius (Acari: Tetranychidae). New Zealand Journal of Zoology 27, 4144.

Plaut, H.N., 1965. On the phenology and control value of Stethorus punctillum Weise as a predator of Tetranychus cinnabarinus Boisd, in Israel. Entomophaga 10, 133137

Pöhle, V.J., Jäckel, B., Plate, H.-P., 2002. Untersuchungsergebnisse zur Spinnmilbenbekämpfung in der Innenraumbegrünung und unter Glas mit Stethorus punctillum (Weise). Gesunde Pflanzen 54, 227-234.

Pollock, D.A., Michels, G.J., 2002. Distributions of Stethorus nigripes Kapur (Coleoptera: Coccinellidae), a predator of Banks grass mite (Oligonychus praetensis (Banks) in the southern United States. Southwestern Entomologist 27, 217-220.

Pollock, D.A., Michels, G.J., 2003. First records of Stethorus histrio Chazeau (Coleoptera: Coccinellidae) from the United States. Southwestern Entomologist 28, 221-222.

Pollock, D.A., Michels, G.J., 2007. Bionomics and distribution of Stethorus caseyi Gordon \& Chapin (Coleoptera: Coccinellidae), with description of the mature larvae. Southwestern Entomologist 32, 143-147.

Prado, E., 1991. Artrópodos y sus enemigos naturales asociados a plantas cultivadas en Chile. Boletín Instituto de Investigaciones Agropecuarias, Estacion Experimental La Platina, Santiago, Chile, 207 pp.

Pratt, P.D., Coombs, E.M., Croft, B.A., 2003. Predation by phytoseiid mites on Tetranychus lintearius (Acari: Tetranychidae), an established weed biological control agent of gorse (Ulex europaeus). Biological Control 26, 40-47.

Putman, W.L., 1955a. The bionomics of Stethorus punctillum Wiese (Coleoptera: Coccinellidae) in Ontario. The Canadian Entomologist 87, 9-33.

Putman, W.L., 1955b. Immature stages of Stethorus punctillum Weise (Coleoptera: Coccinellidae). The Canadian Entomologist 87, 506-508.

Putman, W.L., 1963. Nectar of peach leaf glands as insect food. The Canadian Entomologist 95, 108-109.

Putman, W.L., Herne, D.C., 1966. The role of predators and other biotic agents in regulating the population density of phytophagous mites in Ontario peach orchards. The Canadian Entomologist 98, 808-820.

Puttaswamy, ChannaBasavanna, G.P., 1977. Biology of Stethorus pauperculus Weise (Coleoptera: Coccinellidae), a predator of mites. Mysore Journal of Agricultural Sciences 11, 81-89.

Puttaswamy, ChannaBasavanna, G.P., 1980. Influence of weather factors and predators on the populations of the spidermite Tetranychus ludeni (Acari: Tetranychidae). Indian Journal of Acarology 5, 69-79.

Puttaswamy, Rangaswamy, H.R., 1976. Stethorus keralicus Kapur (Coleoptera: Coccinellidae), a predator of the areca palm mite. Current Research 5, 2728.

Rajotte, E.G., Hull, L.A., Heinemann, P.H., 1987. APPLEMITE: an expert system for European red mite management. Pennsylvania Fruit News 66, 8-16.

Raros, E.S., Haramoto, F.H., 1974. Biology of Stethorus siphonulus Kapur (Coccinellidae: Coleoptera), a predator of spider mites, in Hawaii. Proceedings of the Hawaiian Entomological Society 21, 457-465.

Raworth, D.A., 2001. Development, larval voracity, and greenhouse releases of Stethorus punctillum (Coleoptera: Coccinellidae). The Canadian Entomologist 133, 721-724.

Raworth, D.A., Gillespie, D.R., Roy, M., Thistlewood, H.M.A., 2002. Tetranychus urticae Koch, twospotted spider mite (Acari: Tetranychidae). In: Mason, P.G., Huber, J.T. (Eds.), Biological Control Programmes in Canada, 1981-2000. CABI Publishing, Wallingford, UK, pp. 259-265.

Readshaw, J.L., 1971. An ecological approach to the control of mites in Australian orchards. Journal of the Australian Institute for Agricultural Science 37, 226230.

Readshaw, J.L., 1975. The ecology of tetranychid mites in Australia orchards. Journal of Applied Ecology 12, 473-495.

Richardson, N.L., 1977. The biology of Stethorus loxtoni Britton and Lee (Coleoptera: Coccinellidae) and its potential as a predator of Tetranychus urticae Koch (Acari: Tetranychidae) in California. Ph.D. dissertation, University of California, Berkeley.

Riddick, E.W., Cottrell, T.E., Kidd, K.A., 2009. Natural enemies of the Coccinellidae: Parasites, pathogens, and parasitoids. Biological Control 51, 306-312.

Robinson, A.B., 1953. Notes on Stethorus punctum (Lec.) (Coleoptera: Coccinellidae), a predator of tetranychid mites in Manitoba. In: Proceedings of the 83rd Annual Report of the Entomological Society of Ontario 1952, 24-26.

Rondon, S.I., Cantliffe, D.J., Price, J.F., 2004. The feeding behavior of the bigeyed bug, minute pirate bug, and pink spotted lady beetle relative to main strawberry pests. Environmental Entomologist 33, 1014-1019.

Rosenheim, J.A., Glik, T.E., Goeriz, R.E., Ramert, B., 2004a. Linking a predator's foraging behavior with its effects on herbivore population suppression. Ecology $85,3362-3372$

Rosenheim, J.A., Limburg, D.D., Colfer, R.G., Fournier, V., Hsu, C.L., Leonardo, T.E., Nelson, E.H., 2004b. Herbivore population suppression by an intermediate predator, Phytoseiulus macropilis, is insensitive to the presence of an intraguild predator: an advantage of small body size? Oecologia 140, 577-585.

Rott, A.S., Ponsonby, D.J., 2000. Improving the control of Tetranychus urticae on edible glasshouse crops using a specialist coccinellid (Stethorus punctillum Weise) and a generalist mite (Amblyseius californicus McGregor) as biological control agents. Biocontrol Science and Technology 10, 487-498.

Roy, M., Brodeur, J., Cloutier, C., 2002. Relationship between temperature and development rate of Stethorus punctillum (Coleoptera: Coccinellidae) and its prey Tetranychus mcdanieli (Acarina: Tetranychidae). Environmental Entomology 31, 177-187.

Roy, M., Brodeur, J., Cloutier, C., 2003. Effect of temperature on intrinsic rates of natural increase $\left(r_{m}\right)$ of a coccinellid and its spider mite prey. BioControl $48,57-$ 72.

Roy, M., Brodeur, J., Cloutier, C., 2005. Seasonal activity of the spider mite predators Stethorus punctillum (Coleoptera: Coccinellidae) and Neoseiulus fallacis (Acarina: Phytoseiidae) in raspberry, two predators of Tetranychus mcdanieli (Acarina: Tetranychidae). Biological Control 34, 47-57.

Sabelis, M.W., 1985a. Reproductive strategies. In: Helle, W., Sabelis, M.W., (Eds.), Spider Mites: Their Biology, Natural Enemies and Control, vol. A. Elsevier Amsterdam, pp. 265-278.

Sabelis, M.W., 1985b. Capacity for population increase. In: Helle, W., Sabelis, M.W. (Eds.), Spider Mites: Their Biology, Natural Enemies and Control, vol. B. Elsevier, Amsterdam, pp. 35-41.

Sabelis, M.W., 1991. Life-history evolution in spider mites. In: Schuster, R., Murphy P.W. (Eds.), The Acari: Reproduction, Development and Life-History Strategies. Chapman and Hall, Amsterdam, pp. 23-49.

Sarmah, M., Bhattacharyya, B., 2002. Biology and feeding potential of Stethorus gilvifrons Mulsant (Coccinellidae: Coleoptera) on tea red spider mite, Oligonychus coffeae Neitner. Shashpa 9, 23-26.

Schroeder, D., Zwölfer H., 1970. Studies on insects associated with gorse, Ulex europaeus L. In: Simmonds, F.J. (Ed.), Proceedings of the 1st International Symposium on Biological Control of Weeds, Delémont, Switzerland, pp. 5558.

Scriven, G.T., Fleschner, C.A., 1960. Insectary production of Stethorus species. Journal of Economic Entomology 53, 982-985.

Scriven, G.T., McMurtry, J.A., 1971. Quantitative production and processing of tetranychid mites for large-scale testing or predator production. Journal of Economic Entomology 64, 1255-1257.

Seagraves, M.P., 2009. Lady beetle oviposition behavior in response to the trophic environment. Biological Control 51, 313-322.

Shih, C.I.T., Lin, P.J., Chang, T.W., 1991. Biology, predation, life table and intrinsic rate of increase of Stethorus loi Sasaji. Plant Protection Bulletin (Taipei) 33, 290-300 (in Chinese with English abstract).

Silva, L.D., Bonani, J.P., 2008. Ocorrência de Stethorus (Stethorus) minutalus Gordon \& Chapin (Coleoptera: Coccinellidae) predando Bemisia tabaci biótipo B (Hemiptera: Aleyrodidae) em algodoeiro no Brasil. Neotropical Entomology 37, 86-88.

Ślipiński, A., 2007. Australian Ladybird Beetles (Coleoptera: Coccinellidae) their biology and classification. ABRS, Canberra, $286 \mathrm{pp}$

Smirnoff, W.A., 1958. An artificial diet for rearing coccinellid beetles. The Canadian Entomologist 90, 563-565.

Sohrabi, F., Shishenbor, P., 2007. Functional and numerical responses of Stethorus gilvifrons Mulsant feeding on strawberry spider mite, Tetranychus turkestani Ugarov and Nikolski. Pakistan Journal of Biological Sciences 10, 4563-4566.

Sun, X., Barrett, B.A., Biddinger, D.J., 2000. Changes in fecundity and fertility reductions in adult leafrollers exposed to surfaces treated with the ecdysteroid agonists tebufenozide and methoxyfenozide. Entomologia Experimentalis et Applicata 94, 75-83.

Svendsen, M.S., Hansen, E.W., 2002. Biological and integrated control in ornamentals: successes and challenges. International Organization for Biological Control Western Palearctic Regional Section Bulletin 25, 261-264.

Swaine, G., 1971. Agricultural zoology in Fiji. Great Britain Ministry of Overseas Development. Overseas Research Publication 18. London, H.M. Stationery Office.

Swezey, O.H., 1923. The Hawaiian Planter's Record, vol. 27, pp. 4-7.

Swezey, O.H., 1925. The Hawaiian Planter's Record, vol. 29, pp. 369-376.

Takahashi, H., Takafuji, A., Takabayashi, J., 2001a. Efficiency of a sticky trap for detecting the natural enemy fauna of spider mites (Tetranychidae). Japanese Journal of Applied Entomology and Zoology 45, 189-194 (in Japanese).

Takahashi, H., Takafuiji, A., Takabayashi, J., Yano, S., Shimoda, T., 2001b. Seasonal occurrence of specialist and generalist insect predators of spider mites and their responses to volatiles from spider-mite-infested plants in Japanese pear orchards. Experimental and Applied Acarology 25, 393-402.

Taleb, M.A., Sardar, M.A., 2007. Predation efficiency of insect predators in suppressing red mite, Tetranychus bioculatus attacking marigold. Journal of Agriculture and Rural Development (Gazipur) 5, 98-104.

Tanigoshi, L.K., McMurtry, J.A., 1977. The dynamics of predation of Stethorus picipes (Coleoptera: Coccinellidae) and Typhlodromus floridanus on the prey Oligonychus punicae (Acarina: Phytoseiidae, Tetranychidae). Hilgardia 45, 237-288.

Tanigoshi, L.K., Hoyt, S.C., Croft, B.A., 1983. Basic biology and management components for mite pests and their natural enemies. In: Croft, B.A., Hoyt S.C. (Eds.), Integrated Management of Insect Pests of Pome and Stone Fruits. Wiley Interscience, New York, pp. 153-202.

Tedders, W.L., 1983. Insect management in deciduous orchard ecosystems: habitat manipulation. Environmental Management 7, 29-34.

Thomas, W.P., Burnip, G., 1984. Pests of black currants and the influence of azinphos-methyl on two spotted spider mite. In: Hartley, M.J., Popay, A.J., Popay, A.I. (Eds.), Proceedings of the 37th New Zealand Weed and Pest Control Conference, Christchurch, August 14-16, 1984, pp. 224-227.

Tian, M., 1995. Analysis on control effects of two natural enemies Amblyseius nicholsi and Stethorus punctillum on Panonychus citris by removing method. Chinese Journal of Biocontrol 11, 153-155 (in Chinese).

Travis, J.W., Rajotte, R., Bankert, R., Hickey, K.D., Hull, L.A., Eby, V., Heinamann, P.H., Crassweller, R., McClure, J., Bowser, T., Laughland, D., 1992. A working 
description of the Penn State Apple Orchard Consultant, an expert system. Plant Disease 76, 545-554.

Tsuchiya, M., 2005. The control effect by indigenous natural enemies on citrus red mite (Panonychus citri) on satsumi mandarin (Citru unshiu) groves in summer. Bulletin of the Shizuoka Prefectural Citrus Experiment Station (Japan) 34, 15-27.

Ullah, I., 2000. Aspects of the biology of the ladybird beetle Stethorus vagans (Blackburn) (Coleoptera: Coccinellidae). Doctoral thesis, Centre For Horticulture and Plant Sciences, University of Western Sydney, Hawkesbury, Richmond, NSW Australia, xvii +, 183 pp.

Walters, P.J., 1974. A method for culturing Stethorus spp. (Coleoptera: Coccinellidae) on Tetranychus urticae (Koch) (Acarina: Tetranychidae). Journal of the Australian Entomological Society 13, 245-246.

Walters, P.J., 1976a. Susceptibility of three Stethorus spp. (Coleoptera: Coccinellidae) to selected chemicals used in N.S.W. apple orchards. Journal of the Australian Entomological Society 15, 49-52.

Walters, P.J., 1976b. Effect of five acaricides on Tetranychus urticae (Koch) and its predators, Stethorus spp. (Coleoptera: Coccinellidae) in an apple orchard. Journal of the Australian Entomological Society 15, 53-56.

Walters, P.J., 1976c. Chlordimeform: its prospects for the integrated control of apple pests at Bathurst. Journal of the Australian Entomological Society 15, 57-61.
Wen, H.C., Lee, H.S., 1981. Seasonal occurrence and chemical control of the carmine spider mite (Tetranychus cinnabarinus (Boisduval)) on papaya. Plant Protection Bulletin of Taiwan 23, 131-135 (in Chinese with English summary).

Wen, H.C. Hao, H.H. Lin, C.C., 1993. Seasonal occurrence and chemical control of the citrus red mite (Panonychus citri (McGregor)) on Indian jujube. Journal of Agricultural Research China 42, 303-308 (in Chinese).

Wheeler, A.G., Colburn, R.B., Lehman, R.D., 1973. Stethorus punctillum associated with spruce spider mite on ornamentals. Environmental Entomology 2, 718720 .

Yaseen, M., Bennett, F.D., Ingram, W.R., 1982. Investigations on the cassava mite Mononychellus tanajoa (Bondar) and its natural enemies in the neotropics and East Africa, 1970-1982. In: Cassava Mites, Final Report, Commonwealth Institute for Biological Control, Trinidad, $14 \mathrm{pp}$.

Yigit, A., Uygun, N., 1986. Studies on interactions between hawthorn mite (Tetranychus viennensis Zacher) (Acarina: Tetranychidae) and its predator, Stethorus punctillum Weise (Col.: Coccinellidae). First Turkish National Congress of Biological Control, 406-422.

Yu, G., 1996. A list on Stethorus Weise (Coleoptera: Coccinellidae) from China with description of a new species. Entomotaxonomia 18, 32-36. 